TRANSACTIONS OF THE

AMERICAN MATHEMATICAL SOCIETY

Volume 364, Number 4, April 2012, Pages 1771-1795

S 0002-9947(2011)05355-9

Article electronically published on November 17, 2011

\title{
THE FAILURE OF DIAMOND ON A REFLECTING STATIONARY SET
}

\author{
MOTI GITIK AND ASSAF RINOT
}

\begin{abstract}
It is shown that the failure of $\diamond_{S}$, for a set $S \subseteq \aleph_{\omega+1}$ that reflects stationarily often, is consistent with $\mathrm{GCH}$ and $\mathrm{AP}_{\aleph_{\omega}}$, relative to the existence of a supercompact cardinal. By a theorem of Shelah, GCH and $\square_{\lambda}^{*}$ entails $\nabla_{S}$ for any $S \subseteq \lambda^{+}$that reflects stationarily often.

2. We establish the consistency of existence of a stationary subset of $\left[\aleph_{\omega+1}\right]^{\omega}$ that cannot be thinned out to a stationary set on which the supfunction is injective. This answers a question of König, Larson and Yoshinobu in the negative.

3. We prove that the failure of a diamond-like principle introduced by Džamonja and Shelah is equivalent to the failure of Shelah's strong hypothesis.
\end{abstract}

\section{INTRODUCTION}

0.1. Background. Recall Jensen's diamond principle [10]: for an infinite cardinal $\lambda$ and a stationary set $S \subseteq \lambda^{+}, \nabla_{S}$ asserts the existence of a sequence $\left\langle A_{\delta} \mid \delta \in S\right\rangle$ such that the set $\left\{\delta \in S \mid A \cap \delta=A_{\delta}\right\}$ is stationary for all $A \subseteq \lambda^{+}$.

It is easy to see that $\nabla_{\lambda^{+}}$implies $2^{\lambda}=\lambda^{+}$, and hence it is natural to ask whether the converse holds. Jensen proved that for $\lambda=\aleph_{0}$, the inverse implication fails (see 2). However, for $\lambda>\aleph_{0}$, a recent theorem of Shelah 19 indeed establishes the inverse implication, and moreover, it is proved that $2^{\lambda}=\lambda^{+}$entails $\nabla_{S}$ for every stationary $S$ which is a subset of $E_{\neq \operatorname{cf}(\lambda)}^{\lambda^{+}}:=\left\{\alpha<\lambda^{+} \mid \operatorname{cf}(\alpha) \neq \operatorname{cf}(\lambda)\right\}$.

The result of [19] is optimal: by a theorem of Shelah from [20], GCH is consistent with the failure of $\diamond_{E^{\lambda^{+}}}$for a regular uncountable cardinal $\lambda$. By another theorem of Shelah, from [17, $\S 2$ ], GCH is consistent with the failure of $\diamond_{S}$ for a singular cardinal $\lambda$ and a stationary set $S \subseteq E_{\mathrm{cf}(\lambda)}^{\lambda^{+}}$. However, the latter happens to be a proper subset of $E_{\mathrm{cf}(\lambda)}^{\lambda^{+}}$; more specifically, it is a non-reflecting stationary subset. This leads to the following question:

Question. Suppose $\lambda$ is a singular cardinal and $2^{\lambda}=\lambda^{+}$.

Must $\diamond_{S}$ hold for every $S \subseteq E_{\operatorname{cf}(\lambda)}^{\lambda^{+}}$that reflects stationarily often:1

In [17, §3], Shelah answered the above question in the affirmative, provided that $\square_{\lambda}^{*}$ holds and that $\lambda$ is a strong limit. Later, Zeman 21] applied ideas from [19] and eliminated the "strong limit" hypothesis. Then, in [15], the second author

Received by the editors May 20, 2009 and, in revised form, March 29, 2010.

2010 Mathematics Subject Classification. Primary 03E35; Secondary 03E04, 03 E05.

Key words and phrases. Diamond, sap, square, approachability, very good scale, reflection.

This research was supported by the Israel Science Foundation (grant No. 234/08). The authors would like to thank the referee for his comments and corrections.

${ }^{1}$ We say that $S$ reflects stationarily often iff there are stationarily many $\alpha<\lambda^{+}$with $\operatorname{cf}(\alpha)>\omega$ such that $S \cap \alpha$ is stationary.

(C) 2011 American Mathematical Society Reverts to public domain 28 years from publication 
introduced the Stationary Approachability Property at $\lambda$, abbreviated $\mathrm{SAP}_{\lambda}$, proved that $\mathrm{SAP}_{\lambda}$ is strictly weaker than $\square_{\lambda}^{*}$, and answered the above question positively in the presence of $\mathrm{SAP}_{\lambda}$. It was unknown whether the hypothesis $\mathrm{SAP}_{\lambda}$ can be eliminated or even whether it is possible to replace it with the usual Approachability Property, $\mathrm{AP}_{\lambda}$.

In the present paper we answer the discussed question in the negative, and moreover, do so in the presence of $\mathrm{AP}_{\lambda}$. Let $\operatorname{Refl}_{\lambda}$ denote the assertion that every stationary subset of $E_{\mathrm{cf}(\lambda)}^{\lambda^{+}}$reflects stationarily often; then the main result of this paper reads as follows.

Theorem A. It is relatively consistent with the existence of a supercompact cardinal that all of the following hold simultaneously:

(1) $\mathrm{GCH}$;

(2) $\mathrm{AP}_{\aleph_{\omega}}$;

(3) $\operatorname{Refl}_{\aleph_{\omega}}$;

(4) $\diamond_{S}$ fails for some stationary $S \subseteq E_{\omega}^{\aleph_{\omega+1}}$.

Combining the preceding theorem with the results from [15], we now obtain a complete picture of the effect of weak square principles on diamond 2

Corollary (first three items are from [15]). For a singular cardinal $\lambda$ :

(1) $\mathrm{GCH}+\operatorname{Refl}_{\lambda}+\square_{\lambda}^{*} \Rightarrow \diamond_{\lambda^{+}}^{*}$;

(2) $\mathrm{GCH}+\operatorname{Refl}_{\lambda}+\mathrm{SAP}_{\lambda} \nRightarrow \diamond_{\lambda^{+}}^{*}$;

(3) $\mathrm{GCH}+\mathrm{Refl}_{\lambda}+\mathrm{SAP}_{\lambda} \Rightarrow \diamond_{S}$ for every stationary $S \subseteq \lambda^{+}$;

(4) $\mathrm{GCH}+\operatorname{Refl}_{\lambda}+\mathrm{AP}_{\lambda} \nRightarrow \nabla_{S}$ for every stationary $S \subseteq \lambda^{+}$.

Once that the effect of weak square principles to diamond is well understood, it is natural to study which of the other combinatorial principles from [3, §4] is strong enough to impose an affirmative answer to Question 1. It turns out that even the strongest among these principles does not suffice. We prove:

Theorem B. It is relatively consistent with the existence of a supercompact cardinal that there exists a singular cardinal $\lambda$ for which all of the following hold simultaneously:

(1) $\lambda$ is a strong limit and $2^{\lambda}=\lambda^{+}$;

(2) there exists a very good scale for $\lambda$;

$(3) \nabla_{S}$ fails for some $S \subseteq E_{\mathrm{cf}(\lambda)}^{\lambda^{+}}$that reflects stationarily often.

To conclude the introduction, let us say a few words about the structure of the proof of Theorem A. We start with a supercompact $\kappa$ and a singular cardinal $\lambda$ above it, namely $\lambda:=\kappa^{+\omega}$. We add a generic stationary subset $S \subseteq E_{\mathrm{cf}(\lambda)}^{\lambda^{+}}$and then kill $\nabla_{S}$ by iteration, while preserving the stationarity of $S$ and the supercompactness of $\kappa$. Since $\kappa$ remains supercompact, Refl $\lambda_{\lambda}$ holds, so this already gives an example of a model of GCH on which $\nabla_{S}$ fails for some stationary $S \subseteq E_{\operatorname{cf}(\lambda)}^{\lambda^{+}}$that reflects stationarily often. Nevertheless, by $\operatorname{cf}(\lambda)<\kappa<\lambda$ and a theorem of Shelah from [16], $\mathrm{AP}_{\lambda}$ fails in our model. For this, at the final stage of the proof, we move everything down to $\aleph_{\omega}$ and use a method of Foreman and Magidor from [7] to ensure $\mathrm{AP}_{\aleph_{\omega}}$.

\footnotetext{
${ }^{2}$ For the definition of $\diamond_{\lambda^{+}}^{*}$, as well as Kunen's theorem that $\diamond_{\lambda^{+}}^{*} \Rightarrow \diamond_{S}$ for every stationary $S \subseteq \lambda^{+}$, see $[12$.
} 
The main problem that we address here is an iteration over the successor of a singular. More specifically, the main body of our proof is the argument that $S$ remains stationary after the iteration for killing all diamond sequences over it.

0.2. Organization of this paper. In Section 1, we present a $\lambda^{+}$-directed-closed $\lambda^{++}$-c.c. notion of forcing for introducing a stationary subset of $\lambda^{+}$on which diamond fails. Then, in the presence of a supercompact cardinal, we appeal to this notion of forcing and construct three models in which diamond fails on a set that reflects stationarily often. In particular, Theorem A and Theorem B are proved in this section.

In Section 2, we revisit a theorem by Džamonja and Shelah from [4] in which, starting with a supercompact cardinal, they construct a model satisfying the failure of one of the consequences of diamond. Here, we establish that this particular consequence of diamond is quite weak. We do so by reducing the consistency strength of its failure to the level of existence of a measurable cardinal $\kappa$ of Mitchell order $\kappa^{++}$. In particular, the strength of its failure is lower than that of the failure of a weak square.

In Section 3, we answer a question by König, Larson and Yoshinobu from [11, concerning stationary subsets of $\left[\lambda^{+}\right]^{\omega}$. We do so by linking between the diamond principle and the behavior of the sup-function on generalized stationary sets.

In Section 4, we state a couple of open problems.

0.3. Notation. Generally speaking, we follow the notation and presentation of 12 ] and [3. Let us quickly review our less standard conventions. For cardinals $\kappa<\lambda$, denote $E_{\kappa}^{\lambda}:=\{\alpha<\lambda \mid \operatorname{cf}(\alpha)=\kappa\}$ and $[\lambda]^{\kappa}:=\{X \subseteq \lambda|| X \mid=\kappa\} . E_{>\kappa}^{\lambda}$ and $[\lambda]^{<\kappa}$ are defined analogously. We let Ord denote the class of ordinals. For sets of ordinals $a, b$, we write $a \sqsubseteq b$ or $b \sqsupseteq a$ iff $a$ is an initial segment of $b$, that is, iff $a \subseteq b$ and $b \cap \sup (a)=a \cap \sup (a)$. Given a set of ordinals, $a$, we let $\operatorname{cl}(a):=\{\sup (a \cap \alpha) \mid \alpha \in$ Ord $\}$ denote its topological closure. For $Z \subseteq$ Ord and distinct functions $g, h \in{ }^{Z} 2$, we let $\Delta(g, h):=\min \{\alpha \in Z \mid g(\alpha) \neq h(\alpha)\}$. For $\gamma \in$ Ord and a function $s: \gamma \rightarrow 2$, we denote $\underline{s}:=\{\delta \in \operatorname{dom}(s) \mid s(\delta)=1\}$. For ordinals $\beta \leq \gamma$, we designate the (possibly empty) open interval $((\beta, \gamma))=\{\alpha \in \gamma \mid \beta \in \alpha\}$.

Our forcing conventions are as follows. We denote by $p \geq q$ the fact that $p$ is stronger than $q$. For a ground model set, $x$, we denote its canonical name by $\check{x}$. For arbitrary sets of the generic extension, we designate names such as $\dot{a}$ and $\underset{\sim}{a}$. If $\left\langle p_{1}, \dot{q_{1}}\right\rangle,\left\langle p_{2}, \dot{q_{2}}\right\rangle$ are conditions of a two-step iteration $\mathbb{P} * \mathbb{Q}$ and if $p_{2} \geq p_{1} \Vdash_{\mathbb{P}} \dot{q_{1}} \cong \dot{q}_{2}$, then we slightly abuse notation by writing $\left\langle p_{2}, \dot{q}_{1}\right\rangle=\left\langle p_{2}, \dot{q}_{2}\right\rangle$.

\section{NegAtion OF DiAmond}

1.1. Forcing the failure of diamond. In this subsection, we present for a limit uncountable cardinal $\lambda$, a $\lambda^{+}$-directed-closed, $\lambda^{++}$-c.c., notion of forcing for introducing a stationary subset $S \subseteq \lambda^{+}$on which diamond fails. For simplicity, we shall be focusing on the case $\operatorname{cf}(\lambda)=\omega$. The general case is discussed in subsection 1.3 ,

Definition 1.1. For a cardinal $\lambda$, we define the forcing notion $\mathbb{S}\left(\lambda^{+}\right)$.

A condition $s$ is in $\mathbb{S}\left(\lambda^{+}\right)$iff $s: \gamma \rightarrow 2$ is a function such that $\gamma<\lambda^{+}$and $\underline{s} \subseteq E_{\omega}^{\gamma}$. A condition $s^{\prime}$ is stronger than $s$, denoted $s^{\prime} \geq s$, iff $s^{\prime} \supseteq s$.

The following is obvious. 
Lemma 1.2. For every infinite cardinal $\lambda$ :

(1) $\mathbb{S}\left(\lambda^{+}\right)$has the $\left(2^{\lambda}\right)^{+}$-c.c.;

(2) $\mathbb{S}\left(\lambda^{+}\right)$is $\lambda^{+}$-directed-closed;

(3) every increasing sequence of conditions in $\mathbb{S}\left(\lambda^{+}\right)$of length $<\lambda^{+}$has a least upper bound.

For future needs, it is useful to introduce the following set-name:

$$
\dot{S}\left(\lambda^{+}\right):=\left\{\langle\check{\delta}, s\rangle \mid s \in \mathbb{S}\left(\lambda^{+}\right) \& \delta \in \underline{s}\right\} .
$$

Clearly, if $G$ is $\mathbb{S}\left(\lambda^{+}\right)$-generic, then $\dot{S}\left(\lambda^{+}\right)$is a name for the set $\bigcup\{\underline{s} \mid s \in G\}$. We now consider a natural forcing notion for Killing a given Diamond sequence.

Definition 1.3. For a set $S \subseteq$ Ord and a sequence of sets $\vec{A}$ with $\operatorname{dom}(\vec{A})=S$, we define the forcing notion $\mathbb{K} \mathbb{D}(S, \vec{A})$.

A condition $p$ is in $\mathbb{K D}(S, \vec{A})$ iff $p=(x, c)$, where $c$ is a closed set of ordinals, $x \subseteq \max (c)<\sup (S)$, and $x \cap \delta \neq \vec{A}(\delta)$ for all $\delta \in c \cap S$.

A condition $(x, c)$ is stronger than $\left(x^{\prime}, c^{\prime}\right)$, denoted $(x, c) \geq\left(x^{\prime}, c^{\prime}\right)$, iff $x \sqsupseteq x^{\prime}$ and $c \sqsupseteq c^{\prime}$.

Notice that indeed if $\vec{A}=\left\langle A_{\delta} \mid \delta \in S\right\rangle$ is a $\nabla_{S}$ sequence in $V$, then $\vec{A}$ will cease to be so in $V^{\mathbb{K R} \mathbb{D}(S, \vec{A})}$.

For a strong limit singular cardinal $\lambda$ with $2^{\lambda}=\lambda^{+}$and a subset $S \subseteq \lambda^{+}$, let $\mathbb{K} \mathbb{A D}(S)$ denote the forcing notion for Killing All Diamond sequences over $S$. That is, $\mathbb{K} \mathbb{A D}(S)$ is a $(\leq \lambda)$-support iteration

$$
\left(\left\langle\mathbb{P}_{\alpha} \mid \alpha \leq \lambda^{++}\right\rangle,\left\langle\dot{\mathbb{R}}_{\alpha} \mid \alpha<\lambda^{++}\right\rangle\right)
$$

such that $\mathbb{P}_{0}$ is a trivial forcing, and for all $\alpha<\lambda^{++}, \mathbb{P}_{\alpha}$ forces that $\mathbb{R}_{\alpha}$ is a name for the forcing $\mathbb{K} \mathbb{D}\left(\check{S}, \underset{\sim}{A_{\alpha}}\right)$, whereas $\underset{\sim}{\sim}$ is a $\mathbb{P}_{\alpha}$-name for a sequence chosen by a book-keeping function in such a way that all potential diamond sequences are handled at some stage. The existence of such a function follows from the cardinal arithmetic hypothesis and the $\lambda^{++}$-c.c. of $\mathbb{K} \mathbb{D}(S, \vec{A})$.

Definition 1.4. Let $\mathbb{Q}\left(\lambda^{+}\right):=\mathbb{S}\left(\lambda^{+}\right) * \mathbb{K} \mathbb{A D}\left(\dot{S}\left(\lambda^{+}\right)\right)$.

We also define $\mathbb{Q}^{\prime}\left(\lambda^{+}\right)$. A condition $\langle s, k\rangle$ is in $\mathbb{Q}^{\prime}\left(\lambda^{+}\right)$iff all of the following hold:

(1) $\langle s, k\rangle \in \mathbb{Q}\left(\lambda^{+}\right)$;

(2) $s$ decides the support of $k$ to be, $\operatorname{say}, \operatorname{supp}(k)$;

(3) for all $\alpha \in \operatorname{supp}(k), k(\alpha)$ is an $\mathbb{S}\left(\lambda^{+}\right) * \mathbb{P}_{\alpha}$-canonical name for a pair $\left(x_{\alpha}^{k}, c_{\alpha}^{k}\right)$ 草

(4) for all $\alpha \in \operatorname{supp}(k)$ and $\delta \in \underline{s} \cap c_{\alpha}^{k},\langle s, k\rangle \mid \mathbb{S}\left(\lambda^{+}\right) * \mathbb{P}_{\alpha}$ decides $\overrightarrow{A_{\alpha}}(\delta)$;

(5) $\max \left(c_{\alpha}^{k}\right)=\sup \left(x_{\alpha}^{k}\right)=\sup (\operatorname{dom}(s))$ for all $\alpha \in \operatorname{supp}(k)$.

Lemma 1.5. Every increasing sequence of conditions in $\mathbb{Q}^{\prime}\left(\lambda^{+}\right)$of length $<\lambda^{+}$ has a least upper bound (within $\mathbb{Q}^{\prime}\left(\lambda^{+}\right)$).

Proof. Suppose $\left\langle\left\langle s_{\beta}, k_{\beta}\right\rangle \mid \beta<\theta\right\rangle$ is an increasing sequence of conditions in $\mathbb{Q}^{\prime}\left(\lambda^{+}\right)$, with $\theta<\lambda^{+}$.

Put $\bar{s}:=\bigcup_{\beta<\theta} s_{\beta}$ and $\gamma:=\sup (\operatorname{dom}(\bar{s}))$. Now, define $\langle s, k\rangle$ by letting:

- $s:=\bar{s} \cup\{(\gamma, 0)\}$ if $\gamma \notin \operatorname{dom}(\bar{s})$; otherwise, let $s:=\bar{s}$;

- $s$ decides the support of $k$ to be $\bigcup_{\beta<\theta} \operatorname{supp}\left(k_{\beta}\right)$;

\footnotetext{
${ }^{3}$ In particular, $x_{\alpha}^{k}$ and $c_{\alpha}^{k}$ are elements of the ground model.
} 
- for all $\alpha \in \operatorname{supp}(k)$ :

$$
\begin{gathered}
x_{\alpha}^{k}:=\bigcup\left\{x_{\alpha}^{k_{\beta}} \mid \beta<\theta \& \alpha \in \operatorname{supp}\left(k_{\beta}\right)\right\}, \\
c_{\alpha}^{k}:=\operatorname{cl}\left(\bigcup\left\{c_{\alpha}^{k_{\beta}} \mid \beta<\theta \& \alpha \in \operatorname{supp}\left(k_{\beta}\right)\right\}\right) .
\end{gathered}
$$

To establish that $\langle s, k\rangle \in \mathbb{Q}^{\prime}\left(\lambda^{+}\right)$, let us show that $\langle s, k\rangle \in \mathbb{Q}\left(\lambda^{+}\right)$, namely, that $s \Vdash k \in \mathbb{K} \mathbb{A D}\left(\dot{S}\left(\lambda^{+}\right)\right)$. So, suppose that $t \in \mathbb{S}\left(\lambda^{+}\right), t \geq s, \alpha \in \operatorname{supp}(k)$, and $\delta \in \underline{t} \cap c_{\alpha}^{k}$. Since $\max \left(c_{\alpha}^{k}\right)=\gamma$, we may focus on $t\lceil\gamma+1$. In other words, we may assume that $t=s$. By $\delta \in \underline{s} \cap c_{\alpha}^{k}$ and the definition of $s$, let us pick some $\beta<\theta$ such that $\delta \in s_{\beta}$ and $\alpha \in \operatorname{supp}\left(k_{\beta}\right)$.

Next, by property (5), we have $\max \left(c_{\alpha}^{k_{\beta}}\right)=\sup \left(\operatorname{dom}\left(s_{\beta}\right)\right) \geq \delta$, and hence $\delta \in c_{\alpha}^{k_{\beta}}$. By property $(4),\left\langle s_{\beta}, k_{\beta}\right\rangle \uparrow \mathbb{S}\left(\lambda^{+}\right) * \mathbb{P}_{\alpha}$ decides $\overrightarrow{A_{\alpha}}(\delta)$, and hence $x_{\alpha}^{k_{\beta}} \cap \delta \neq \overrightarrow{A_{\alpha}}(\delta)$. Finally, by property (5), we have $\sup \left(x_{\alpha}^{k_{\beta}}\right)=\max \left(c_{\alpha}^{k_{\beta}}\right) \geq \delta$, and hence $x_{\alpha}^{k_{\beta}} \cap \delta=$ $x_{\alpha}^{k} \cap \delta$, so $x_{\alpha}^{k} \cap \delta \neq \overrightarrow{A_{\alpha}}(\delta)$.

So, $\langle s, k\rangle$ is a legitimate element of $\mathbb{Q}^{\prime}\left(\lambda^{+}\right)$, and it is clearly an upper bound of the above sequence. Finally, notice that if $\langle s, k\rangle$ is not the least upper bound, then $\langle\bar{s} \cup\{(\gamma, 1)\}, k\rangle$ is a legitimate condition as well; hence, in this case, the least upper bound is just $\langle\bar{s}, k\rangle$.

Lemma 1.6. $\mathbb{Q}^{\prime}\left(\lambda^{+}\right)$is $\lambda^{+}$-directed closed.

Proof. Virtually the same proof as the preceding.

Lemma 1.7. Assume $\lambda$ is a strong limit singular cardinal and $2^{\lambda}=\lambda^{+}$. Then:

(1) $\left|\mathbb{Q}\left(\lambda^{+}\right)\right|=\lambda^{++}$

(2) $\mathbb{Q}\left(\lambda^{+}\right)$has the $\lambda^{++}$-c.c.;

(3) $\mathbb{Q}^{\prime}\left(\lambda^{+}\right)$is dense in $\mathbb{Q}\left(\lambda^{+}\right)$.

Proof. (1) is obvious, and (2) follows from a standard $\Delta$-system argument.

(3) To simplify the notation, for all $\beta \leq \lambda^{++}$, let $\mathbb{Q}_{\beta}:=\mathbb{S}\left(\lambda^{+}\right) * \mathbb{P}_{\beta}$ and $\mathbb{Q}_{\beta}^{\prime}:=$ $\mathbb{Q}^{\prime}\left(\lambda^{+}\right) \backslash \mathbb{Q}_{\beta}$. Note that the proof of Lemma 1.5] shows that every increasing sequence of conditions in $\mathbb{Q}_{\beta}^{\prime}$ of length $<\lambda^{+}$has a least upper bound. We now prove by induction on $\beta \leq \lambda^{++}$that $\mathbb{Q}_{\beta}^{\prime}$ is dense in $\mathbb{Q}_{\beta}$.

Induction base: For $\beta=0$, we have $\mathbb{Q}_{\beta}^{\prime}=\mathbb{S}\left(\lambda^{+}\right) * \mathbb{P}_{0}=\mathbb{Q}_{\beta}$.

Induction step: Suppose the claim holds for $\alpha$ and that $\langle s, k\rangle$ is a given element of $\overline{\mathbb{Q}_{\beta}}$, for $\beta=\alpha+1$. We would like to find a condition in $\mathbb{Q}_{\beta}^{\prime}$ which is stronger than $\langle s, k\rangle$.

Since $\mathbb{S}\left(\lambda^{+}\right)$is $\lambda^{+}$-closed and $\mathbb{P}_{\beta}$ is a $(\leq \lambda)$-support iteration, we may assume that $s$ already decides the support of $k$. To avoid trivialities, we may also assume that $\alpha \in \operatorname{supp}(k)$.

Since $\mathbb{Q}_{\alpha}^{\prime}$ is a $\lambda^{+}$-closed, dense subset of $\mathbb{Q}_{\alpha}$ and $k(\alpha)$ is a $\mathbb{Q}_{\alpha}$-name for a pair of bounded subsets of $\lambda^{+}$, let us pick a condition $\left\langle s_{0}, k_{0}\right\rangle \geq\langle s, k\rangle$ and a pair $(x, c)$ such that $\left\langle s_{0}, k_{0}\right\rangle \vdash \mathbb{Q}_{\alpha} \in \mathbb{Q}_{\alpha}^{\prime}, k_{0}(\alpha)$ is the $\mathbb{Q}_{\alpha}$-canonical name for $(x, c)$, and

$$
\left\langle s_{0}, k_{0}\right\rangle \vdash \mathbb{Q}_{\alpha} \Vdash k(\alpha)=k_{0}(\alpha) .
$$

Next, for all $\delta<\lambda^{+}$, let $D_{\delta}$ denote the collection of all conditions $\left\langle s^{\prime}, k^{\prime}\right\rangle \in \mathbb{Q}_{\beta}$ such that all of the following holds

- $\left\langle s^{\prime}, k^{\prime}\right\rangle \geq\left\langle s_{0}, k_{0}\right\rangle$;

- $\left\langle s^{\prime}, k^{\prime}\right\rangle \uparrow \mathbb{Q}_{\alpha} \in \mathbb{Q}_{\alpha}^{\prime}$; 
- $\left\langle s^{\prime}, k^{\prime}\right\rangle \uparrow \mathbb{Q}_{\alpha}$ decides $\overrightarrow{A_{\alpha}}(\delta)$;

- $k^{\prime}(\alpha)=k_{0}(\alpha)$.

Since $\mathbb{Q}_{\alpha}^{\prime}$ is a $\lambda^{+}$-closed, dense subset of $\mathbb{Q}_{\alpha}$, we get that each $D_{\delta}$ is dense in $\mathbb{Q}_{\beta}$ above $\left\langle s_{0}, k_{0}\right\rangle$, so let us pick a condition $\left\langle s_{1}, k_{1}\right\rangle \in \bigcap_{\delta \in c} D_{\delta}$.

Let

$$
\gamma:=\sup \left(x \cup c \cup \operatorname{dom}\left(s_{1}\right) \cup \bigcup\left\{\max \left(c_{i}^{k_{1}}\right) \mid i \in \operatorname{supp}\left(k_{1}\right) \cap \alpha\right\}\right) .
$$

Now, we take the condition $\left\langle s^{*}, k^{*}\right\rangle$ as follows:

- $s^{*}: \gamma+\omega+1 \rightarrow 2$ is the function satisfying for all $\delta \leq \gamma+\omega$,

$$
s^{*}(\delta)= \begin{cases}s(\delta), & \delta \in \operatorname{dom}\left(s_{1}\right), \\ 0, & \text { otherwise }\end{cases}
$$

- $s^{*}$ decides the support of $k^{*}$ to be $\operatorname{supp}\left(k_{1}\right)$;

- for all $i \in \operatorname{supp}\left(k^{*}\right), k^{*}(i)$ is the canonical name for the pair $\left(x_{i}, c_{i}\right)$, where

$$
\left(x_{i}, c_{i}\right):= \begin{cases}\left(x_{i}^{k_{1}} \cup((\gamma, \gamma+\omega)), c_{i}^{k_{1}} \cup\{\gamma+\omega\}\right), & i<\alpha, \\ (x \cup((\gamma, \gamma+\omega)), c \cup\{\gamma+\omega\}), & i=\alpha .\end{cases}
$$

Then $\left\langle s^{*}, k^{*}\right\rangle \geq\left\langle s_{1}, k_{1}\right\rangle \geq\langle s, k\rangle, \sup \left(\operatorname{dom}\left(s^{*}\right)\right)=\max \left(c_{i}^{k^{*}}\right)=\sup \left(x_{i}^{k^{*}}\right)=\gamma+\omega$ for all $i \in \operatorname{supp}\left(k^{*}\right),\left\langle s^{*}, k^{*}\right\rangle \uparrow \mathbb{Q}_{\alpha} \in \mathbb{Q}_{\alpha}^{\prime}$, and $\underline{t} \cap c_{\alpha}^{k^{*}}=s_{1} \cap c$ for every $t \geq s^{*}$, so the fact that $\left\langle s_{1}, k_{1}\right\rangle$ is in $\bigcap_{\delta \in c} D_{\delta}$ entails clause (4) of Definition 1.4. Namely, we have found $\left\langle s^{*}, k^{*}\right\rangle \in \mathbb{Q}_{\beta}^{\prime}$, which is stronger than $\langle s, k\rangle$.

Limit step: Suppose $\beta$ is a limit ordinal and $\langle s, k\rangle \in \mathbb{Q}_{\beta}$.

Clearly, we may assume that $s$ decides the support of $k$. To avoid trivialities, we may also assume that $\sup (\operatorname{supp}(k))=\beta$. In particular, $\operatorname{cf}(\beta)<\lambda$.

Let $\left\langle\beta_{\alpha} \mid \alpha<\operatorname{cf}(\beta)\right\rangle$ be an increasing sequence of ordinals converging to $\beta$. Evidently, this sequence may be chosen in such a way that $\operatorname{cf}\left(\beta_{\alpha}\right)<\lambda$ for all $\alpha \leq \operatorname{cf}(\beta)$, where $\beta_{\operatorname{cf}(\beta)}:=\beta$.

Recursively define an increasing sequence of conditions in $\mathbb{Q}\left(\lambda^{+}\right),\left\langle\left\langle s_{\alpha}, k_{\alpha}\right\rangle\right| \alpha \leq$ $\operatorname{cf}(\beta)\rangle$ in such a way that for all $\alpha \leq \operatorname{cf}(\beta)$ :

- $\left\langle s_{0}, k_{0}\right\rangle=\langle s, k\rangle$;

- $s_{\alpha}$ decides the support of $k_{\alpha}$;

- $\left\langle s_{\alpha}, k_{\alpha}\right\rangle \uparrow \mathbb{Q}_{\beta_{\alpha}} \in \mathbb{Q}_{\beta_{\alpha}}^{\prime}$;

- $k_{\alpha} \uparrow\left(\lambda^{++} \backslash \beta_{\alpha}\right)=k \uparrow\left(\lambda^{++} \backslash \beta_{\alpha}\right)$.

The successor stage simply utilizes the induction hypothesis, so let us show how to handle the limit stage of the recursion. Suppose $\alpha \leq \operatorname{cf}(\beta)$ is a limit ordinal and $\left\langle\left\langle s_{\varepsilon}, k_{\varepsilon}\right\rangle \mid \varepsilon<\alpha\right\rangle$ is defined.

Fix $\varepsilon<\alpha$. By the induction hypothesis, $\left\langle\left\langle s_{\eta}, k_{\eta}\right\rangle \uparrow \mathbb{Q}_{\beta_{\varepsilon}} \mid \varepsilon \leq \eta<\alpha\right\rangle$ is an increasing sequence of conditions in $\mathbb{Q}_{\beta_{\varepsilon}}^{\prime}$ of length $<\lambda^{+}$, so let $q_{\varepsilon}$ denote its least upper bound. Then, for all $\varepsilon<\alpha$, let $\left\langle s^{\varepsilon}, k^{\varepsilon}\right\rangle$ denote the least upper bound of the weakly increasing sequence $\left\langle q_{\eta} \uparrow \mathbb{Q}_{\beta_{\varepsilon}} \mid \varepsilon \leq \eta<\alpha\right\rangle$.

Notice that $\left\langle s^{\varsigma}, k^{\varsigma}\right\rangle \uparrow \mathbb{Q}_{\beta_{\varepsilon}}=\left\langle s^{\epsilon}, k^{\epsilon}\right\rangle \uparrow \mathbb{Q}_{\beta_{\varepsilon}}$ whenever $\varsigma \leq \varepsilon \leq \epsilon<\alpha$.

We now define $\left\langle s_{\alpha}, k_{\alpha}\right\rangle$ and then argue that it is indeed a legitimate condition. Thus, let $\left\langle s_{\alpha}, k_{\alpha}\right\rangle$ be the condition satisfying:

- $s_{\alpha}=s^{0}$;

- $s_{\alpha}$ decides the support of $k_{\alpha}$ to be $\bigcup_{\varepsilon<\alpha} \operatorname{supp}\left(k^{\varepsilon}\right) \cup \operatorname{supp}(k)$; 
- for all $i \in \operatorname{supp}\left(k_{\alpha}\right) \cap \beta_{\alpha}$ :

$$
k_{\alpha}(i)=k^{\varepsilon}(i), \text { where } \varepsilon:=\min \left\{\varepsilon^{\prime} \mid i \in \operatorname{supp}\left(k^{\varepsilon^{\prime}}\right)\right\} ;
$$

- for all $i \in \operatorname{supp}\left(k_{\alpha}\right) \backslash \beta_{\alpha}$ :

$$
k_{\alpha}(i)=k(i) .
$$

Evidently, for all $\varepsilon<\alpha$, we have $\left\langle s_{\alpha}, k_{\alpha}\right\rangle \mid \mathbb{Q}_{\beta_{\varepsilon}}=\left\langle s^{\varepsilon}, k^{\varepsilon}\right\rangle$. Since $\operatorname{cf}\left(\beta_{\alpha}\right)<\lambda$, the forcing $\mathbb{P}_{\beta_{\alpha}}$ is the inverse limit of $\left\langle\mathbb{P}_{\varepsilon} \mid \varepsilon<\beta_{\alpha}\right\rangle$, and hence $\left\langle s_{\alpha}, k_{\alpha}\right\rangle\left\lceil\mathbb{Q}_{\beta_{\alpha}}\right.$, the restriction of our proposed condition, is in $\mathbb{Q}_{\beta_{\alpha}}$. By arguments which, by now, are standard, we moreover have $\left\langle s_{\alpha}, k_{\alpha}\right\rangle \uparrow \mathbb{Q}_{\beta_{\alpha}} \in \mathbb{Q}_{\beta_{\alpha}}^{\prime}$.

Finally, as $\left\langle s_{\alpha}, k_{\alpha}\right\rangle \uparrow \mathbb{Q}_{\beta_{\alpha}} \geq\langle s, k\rangle \uparrow \mathbb{Q}_{\beta_{\alpha}}, k_{\alpha} \uparrow\left(\lambda^{++} \backslash \beta_{\alpha}\right)=k \uparrow\left(\lambda^{++} \backslash \beta_{\alpha}\right)$ and

$$
\langle s, k\rangle \uparrow \mathbb{Q}_{\beta_{\alpha}} \Vdash k \uparrow\left(\lambda^{++} \backslash \beta_{\alpha}\right) \in \mathbb{K} \mathbb{A D}\left(\dot{S}\left(\lambda^{+}\right)\right) \uparrow\left(\lambda^{++} \backslash \beta_{\alpha}\right),
$$

we obtain

$$
\left\langle s_{\alpha}, k_{\alpha}\right\rangle\left\lceil\mathbb{Q}_{\beta_{\alpha}} \Vdash k_{\alpha} \uparrow\left(\lambda^{++} \backslash \beta_{\alpha}\right) \in \mathbb{K} \mathbb{A D}\left(\dot{S}\left(\lambda^{+}\right)\right) \uparrow\left(\lambda^{++} \backslash \beta_{\alpha}\right),\right.
$$

and hence $\left\langle s_{\alpha}, k_{\alpha}\right\rangle$ is a legitimate condition of $\mathbb{Q}\left(\lambda^{+}\right)$. Thus, the recursion may indeed be carried out, and we end up with a condition $\left\langle s_{\operatorname{cf}(\beta)}, k_{\mathrm{cf}(\beta)}\right\rangle \in \mathbb{Q}_{\beta}^{\prime}$ which is stronger than $\langle s, k\rangle$, as requested.

The next theorem is the core of our proof. We encourage the reader to notice the role of the fact that $S$ concentrates on the critical cofinality, i.e., that $S \subseteq E_{\mathrm{cf}(\lambda)}^{\lambda^{+}}$约

Theorem 1.8. Suppose $\lambda>\operatorname{cf}(\lambda)=\omega$ is a strong limit and $2^{\lambda}=\lambda^{+}$.

If $G * H$ is $\mathbb{Q}\left(\lambda^{+}\right)$-generic, then letting $S:=\bigcup G$ we have

$$
V[G][H] \models S \text { is stationary. }
$$

Proof. Fix a name $\dot{E}$ and a condition $\left\langle s^{*}, k^{*}\right\rangle \in \mathbb{Q}^{\prime}\left(\lambda^{+}\right)$forcing that $\dot{E}$ is a club subset of $\lambda^{+}$. Clearly, we may assume that $0 \in \operatorname{supp}\left(k^{*}\right)$ and that $\left|\operatorname{supp}\left(k^{*}\right)\right|=\lambda$.

Thus, our goal is to find an extension of $\left\langle s^{*}, k^{*}\right\rangle$ that forces that $E$ meets $S$. Fix a large enough regular cardinal $\chi$ and an elementary submodel $M \prec\left\langle H(\chi),<_{\chi}\right)$ satisfying:

- $|M|=\lambda$;

- $\lambda<M \cap \lambda^{+} \in E_{\omega}^{\lambda^{+}}$;

- $\left\langle\mathbb{Q}\left(\lambda^{+}\right), \mathbb{Q}^{\prime}\left(\lambda^{+}\right),\left\langle s^{*}, k^{*}\right\rangle, \dot{E}\right\rangle \in M$.

Notice that since $\lambda$ is a strong limit, we have ${ }^{Z} \subseteq M$ for all $Z \in M$ with $|Z|<\lambda$. For a set $Z \in\left[\lambda^{++}\right]^{\lambda}$, let $\psi_{Z}: \lambda \rightarrow Z$ be the $<_{\chi}$-least surjection. Fix $\left\{\lambda_{n} \mid n<\right.$ $\omega\} \in M$ which is a cofinal subset of $\lambda$. Evidently, for every $Z \in\left[\lambda^{++}\right]^{\lambda} \cap M$ and $n<\omega$, we have $\psi_{Z}$ " $\lambda_{n} \in M$.

Definition 1.8.1 (Deciding the club). For $\alpha<\lambda^{+}$and a condition $\langle s, k\rangle \geq\left\langle s^{*}, k^{*}\right\rangle$, let $\langle s, k\rangle^{\alpha}$ denote the $<_{\chi}$-least extension of $\langle s, k\rangle$ such that:

- $\langle s, k\rangle^{\alpha} \in \mathbb{Q}^{\prime}\left(\lambda^{+}\right)$;

- $\langle s, k\rangle^{\alpha}$ decides a value for $\min (E \backslash \alpha)$;

- if $\langle s, k\rangle^{\alpha}=\left\langle s^{\prime}, k^{\prime}\right\rangle$, then for some $\gamma \geq \alpha, \operatorname{dom}\left(s^{\prime}\right)=\gamma+\omega+1$ and $s^{\prime}(\gamma+\omega)=$ 0 .

Notice that if $\langle s, k\rangle \in M$ and $\alpha \in M \cap \lambda^{+}$, then $\langle s, k\rangle^{\alpha} \in M$ as well.

\footnotetext{
${ }^{4}$ Recall that by [19], $2^{\lambda}=\lambda^{+}$entails $\diamond_{S}$ for every $S$ which does not concentrate on the critical cofinality.
} 
Definition 1.8.2 (Branching extensions). For a condition $\langle s, k\rangle \in \mathbb{Q}^{\prime}\left(\lambda^{+}\right)$, a set $Z \in\left[\lambda^{++}\right]^{\leq \lambda}$, a function $g: Z \rightarrow 2$, and an ordinal $\gamma \geq \sup (\operatorname{dom}(s))$, we shall define $\langle s, k\rangle_{\gamma}^{g}$. Let $\langle s, k\rangle_{\gamma}^{g}=\left\langle s^{\prime}, k^{\prime}\right\rangle$ be the $\leq_{\chi}$-least extension of $\langle s, k\rangle$ such that:

- $\left\langle s^{\prime}, k^{\prime}\right\rangle \in \mathbb{Q}^{\prime}\left(\lambda^{+}\right)$

- $s^{\prime}: \gamma+\omega+1 \rightarrow 2$ is the function satisfying for all $\delta \leq \gamma+\omega$ :

$$
s^{\prime}(\delta):= \begin{cases}s(\delta), & \delta \in \operatorname{dom}(s), \\ 0, & \text { otherwise; }\end{cases}
$$

- $\operatorname{supp}\left(k^{\prime}\right)=\operatorname{supp}(k) \cup Z$;

- for all $i \in \operatorname{supp}\left(k^{\prime}\right)$ :

$$
\left(x_{i}^{k^{\prime}}, c_{i}^{k^{\prime}}\right)= \begin{cases}(((\gamma+g(i), \gamma+\omega)),\{\gamma+\omega\}), & i \in Z \backslash \operatorname{supp}(k), \\ \left(x_{i}^{k} \cup((\gamma+g(i), \gamma+\omega)), c_{i}^{k} \cup\{\gamma+\omega\}\right), & i \in Z \cap \operatorname{supp}(k), \\ \left(x_{i}^{k} \cup((\gamma, \gamma+\omega)), c_{i}^{k} \cup\{\gamma+\omega\}\right), & \text { else. }\end{cases}
$$

To see that the definition is good, just notice that for all $t \geq s^{\prime}$ and $i \in \operatorname{supp}\left(k^{\prime}\right)$, if $\underline{t} \cap c_{i}^{k^{\prime}} \neq \emptyset$, then $i \in \operatorname{supp}(k)$, and $x_{i}^{k^{\prime}} \cap \delta=x_{i}^{k} \cap \delta$ for all $\delta \in \underline{t} \cap c_{i}^{k^{\prime}}=\underline{s} \cap c_{i}^{k}$.

Evidently, if $\{g, \gamma,\langle s, k\rangle\} \subseteq M$, then $\langle s, k\rangle_{\gamma}^{g}$ is in $M$ as well.

Definition 1.8.3 (Mixing two conditions). Given $q_{0}=\left\langle s_{0}, k_{0}\right\rangle, q_{1}=\left\langle s_{1}, k_{1}\right\rangle$ in $\mathbb{Q}^{\prime}\left(\lambda^{+}\right)$, and $\beta<\lambda^{++}$such that $\left\langle s_{0}, k_{0}\right\rangle\left\lceil\mathbb{S}\left(\lambda^{+}\right) * \mathbb{P}_{\beta} \leq\left\langle s_{1}, k_{1}\right\rangle\left\lceil\mathbb{S}\left(\lambda^{+}\right) * \mathbb{P}_{\beta}\right.\right.$, let $\operatorname{mix}\left(q_{1}, \beta, q_{0}\right)$ be the $<_{\chi}$-least condition $\left\langle s^{\prime}, k^{\prime}\right\rangle$ such that:

- $\left\langle s^{\prime}, k^{\prime}\right\rangle \in \mathbb{Q}^{\prime}\left(\lambda^{+}\right)$;

- if $\gamma<\lambda^{+}$is the minimal ordinal such that there exists a function $s^{\prime}$ : $\gamma+\omega+1 \rightarrow 2$ with $s^{\prime} \supseteq s_{1}$ and $s^{\prime}(\gamma+\omega)=0$, then $s^{\prime}$ is a witness to that;

- $\operatorname{supp}\left(k^{\prime}\right)=\left(\operatorname{supp}\left(k_{1}\right) \cap \beta\right) \cup \operatorname{supp}\left(k_{0}\right)$;

- for all $i \in \operatorname{supp}\left(k^{\prime}\right)$ :

$$
\left(x_{i}^{k^{\prime}}, c_{i}^{k^{\prime}}\right)= \begin{cases}\left(x_{i}^{k_{1}} \cup\left(\left(\max \left(c_{i}^{k_{1}}\right), \gamma+\omega\right)\right), c_{i}^{k_{1}} \cup\{\gamma+\omega\}\right), & i<\beta, \\ \left(x_{i}^{k_{0}} \cup\left(\left(\max \left(c_{i}^{k_{0}}\right), \gamma+\omega\right)\right), c_{i}^{k_{0}} \cup\{\gamma+\omega\}\right), & \text { else. }\end{cases}
$$

It is not hard to see that the definition is good and that $\operatorname{mix}\left(q_{1}, \beta, q_{0}\right) \in M$ whenever $\left\{q_{1}, \beta, q_{0}\right\} \subseteq M$. Notice that $\operatorname{mix}\left(q_{1}, \beta, q_{0}\right)$ depends only on $q_{0}$ and $q_{1} \uparrow$ $\mathbb{S}\left(\lambda^{+}\right) * \mathbb{P}_{\beta}$ and that this notion also makes sense in case that $\beta=0$.

Claim 1.8.4. Suppose $g_{1}, g_{0} \in{ }^{Z} 2$ for a given set $Z \in\left[\lambda^{++}\right] \leq \lambda$ and that $q \in \mathbb{Q}^{\prime}\left(\lambda^{+}\right)$.

If $\beta \leq \Delta\left(g_{0}, g_{1}\right)$ and $\alpha, \gamma<\lambda^{+}$, then $\operatorname{mix}\left(q_{\gamma}^{g_{1}}, \beta, q_{\gamma}^{g_{0}}\right)=q_{\gamma}^{g_{0}}$, and $\operatorname{mix}\left(\left(q_{\gamma}^{g_{1}}\right)^{\alpha}, \beta, q_{\gamma}^{g_{0}}\right)$ is a well-defined extension of $q_{\gamma}^{g_{0}}$.

Proof. This follows immediately from the above definitions, and we encourage the reader to digest these definitions by verifying this claim.

Put $\tau:=M \cap \lambda^{+}$and pick a countable set of limit ordinals $\left\{\tau_{n} \mid n<\omega\right\} \subseteq M \cap \lambda^{+}$ with $\sup _{n<\omega} \tau_{n}=\tau$. Denote $Z_{-1}:=\emptyset$. We now recursively define a sequence of elements of $M,\left\langle\left(\gamma_{n}, Y_{n}, Z_{n}, \mathcal{F}_{n}\right) \mid n<\omega\right\rangle$, in such a way that for all $n<\omega$ :

(1) $\gamma_{n}<\lambda^{+}$;

(2) $\left\{Y_{n} \mid n<\omega\right\} \subseteq\left[\lambda^{++}\right]^{\lambda}$ and $\left\{Z_{n} \mid n<\omega\right\} \subseteq\left[\lambda^{++}\right]^{<\lambda}$ are increasing chains, and $\bigcup_{n<\omega} Y_{n}=\bigcup_{n<\omega} Z_{n}$

(3) for every $g \in{ }^{Z_{n}} 2$, we define a condition $q_{g} \in \mathbb{Q}^{\prime}\left(\lambda^{+}\right)$in such a way that $m<n$ implies $q_{g \mid Z_{m}} \leq q_{g}$, and we let $\mathcal{F}_{n}:=\left\{q_{g} \mid g \in{ }^{Z_{n}} 2\right\} ;$ 
(4) for every $g \in{ }^{Z_{n}} 2$, there exists some $r \in \mathbb{Q}^{\prime}\left(\lambda^{+}\right)$such that

$$
q_{g} \geq r_{\gamma_{n}}^{g \uparrow\left(Z_{n} \backslash Z_{n-1}\right)} \geq r \geq\left\langle s^{*}, k^{*}\right\rangle ;
$$

(5) for every $g, h \in Z_{n} 2$ and $\beta<\lambda^{++}$, if $g\lceil\beta=h \uparrow \beta$, then

$$
q_{g} \uparrow \mathbb{S}\left(\lambda^{+}\right) * \mathbb{P}_{\beta}=q_{h} \uparrow \mathbb{S}\left(\lambda^{+}\right) * \mathbb{P}_{\beta}
$$

(6) if $\langle s, k\rangle \in \mathcal{F}_{n}$, then $\langle s, k\rangle$ decides a value for $\min \left(E \backslash \tau_{n}\right)$, and $\operatorname{dom}(s)>\tau_{n}$.

We commence with letting $\gamma_{0}:=\sup \left\{\max \left(c_{i}^{k^{*}}\right) \mid i \in \operatorname{supp}\left(k^{*}\right)\right\}, Y_{0}:=\operatorname{supp}\left(k^{*}\right)$, and $Z_{0}:=\psi_{Y_{0}}$ " $\lambda_{0}$. Next, we would like to define $q_{g}$ for all $g \in{ }^{Z_{0}} 2$. Let $\left\{g_{j} \mid\right.$ $\left.j<2^{\lambda_{0}}\right\}$ be the $<_{\chi}$-least injective enumeration of $Z_{0} 2$. We shall define an upper triangular matrix of conditions, $\left\{q_{j l} \mid j \leq l<2^{\lambda_{0}}\right\}$, in such a way that for all $j \leq j^{\prime} \leq l \leq l^{\prime}<2^{\lambda_{0}}$, the following holds:

(a) $r_{\gamma_{0}}^{g_{j}} \leq q_{j j} \leq q_{j l} \leq q_{j l^{\prime}}$, where $r:=\left\langle s^{*}, k^{*}\right\rangle$;

(b) $p^{\tau_{0}} \leq q_{j j}$ for some condition $p \in \mathbb{Q}^{\prime}\left(\lambda^{+}\right)$;

(c) $q_{j l^{\prime}} \uparrow \mathbb{S}\left(\lambda^{+}\right) * \mathbb{P}_{\beta}=q_{j^{\prime} l^{\prime}}\left\lceil\mathbb{S}\left(\lambda^{+}\right) * \mathbb{P}_{\beta}\right.$ whenever $g_{j}\left\lceil\beta=g_{j^{\prime}} \uparrow \beta\right.$.

Once we have that, for each $g \in{ }^{Z_{0}} 2$ we pick the unique $j$ such that $g_{j}=g$ and let $q_{g}$ be the least upper bound of the increasing sequence $\left\langle q_{j l} \mid j \leq l<2^{\lambda_{0}}\right\rangle$. Then item (a) takes care of requirement (4), item (b) establishes requirement (6), and item (c) yields requirement (5).

Thus, the $j_{t h}$ row of the matrix is responsible for the condition $q_{g_{j}}$. The actual definition of the matrix, however, is obtained along the columns. Namely, we define $\left\{q_{j l} \mid j \leq l<2^{\lambda_{0}}\right\}$ by induction on $l<2^{\lambda_{0}}$.

Induction base: Let $q_{00}:=\left(r_{\gamma_{0}}^{g_{0}}\right)^{\tau_{0}}$.

Successor step: Suppose $l<2^{\lambda_{0}}$ and $\left\{q_{j l} \mid j \leq l\right\}$ has already been defined. We would like to define $\left\{q_{j l^{\prime}} \mid j \leq l^{\prime}\right\}$, for $l^{\prime}:=l+1$.

Put $\alpha:=\sup \left\{\Delta\left(g_{j}, g_{l^{\prime}}\right)+1 \mid j<l^{\prime}\right\}$. For all $\beta<\alpha$, let $j_{\beta}$ be the least such that $j_{\beta}<l^{\prime}$ and $g_{j_{\beta}} \uparrow \beta=g_{l^{\prime}} \uparrow \beta$. Note that if $j<l^{\prime}$ and $\beta=\Delta\left(g_{j}, g_{l^{\prime}}\right)$, then we have $g_{j} \uparrow \beta=g_{j_{\beta}}\lceil\beta$.

Fix $\beta<\alpha$. By Definition 1.8.2, we have $r_{\gamma_{0}}^{g_{j_{\beta}}}\left\lceil\mathbb{S}\left(\lambda^{+}\right) * \mathbb{P}_{\beta}=r_{\gamma_{0}}^{g_{l^{\prime}}} \uparrow \mathbb{S}\left(\lambda^{+}\right) * \mathbb{P}_{\beta}\right.$, and by property (a), we have $r_{\gamma_{0}}^{g_{j_{\beta}}} \leq q_{j_{\beta} l}$. It follows that $r_{\gamma_{0}}^{g_{l^{\prime}}}\left\lceil\mathbb{S}\left(\lambda^{+}\right) * \mathbb{P}_{\beta} \leq q_{j_{\beta} l} \uparrow\right.$ $\mathbb{S}\left(\lambda^{+}\right) * \mathbb{P}_{\beta}$, and hence $\operatorname{mix}\left(q_{j_{\beta} l}, \beta, r_{\gamma_{0}}^{g_{l^{\prime}}}\right)$ is a well-defined extension of $r_{\gamma_{0}}^{g_{l^{\prime}}}$.

By property (c), we get that $\beta<\gamma<\alpha$ implies $q_{j_{\beta} l} \uparrow \mathbb{S}\left(\lambda^{+}\right) * \mathbb{P}_{\beta}=q_{j_{\gamma} l} \uparrow$ $\mathbb{S}\left(\lambda^{+}\right) * \mathbb{P}_{\beta}$ and $\operatorname{mix}\left(q_{j_{\beta} l}, \beta, r_{\gamma_{0}}^{g_{l^{\prime}}}\right)=\operatorname{mix}\left(q_{j_{\gamma} l}, \beta, r_{\gamma_{0}}^{g_{l^{\prime}}}\right) \leq \operatorname{mix}\left(q_{j_{\gamma} l}, \gamma, r_{\gamma_{0}}^{g_{l^{\prime}}}\right)$.

For all $\beta<\alpha$, let $p_{\beta}:=\operatorname{mix}\left(q_{j_{\beta} l}, \beta, r_{\gamma_{0}}^{g_{l^{\prime}}}\right)$. Then, we have just established that $\left\langle p_{\beta} \mid \beta<\alpha\right\rangle$ is an increasing sequence of conditions, with $p_{0} \geq r_{\gamma_{0}}^{g_{l^{\prime}}}$, and $p_{\beta} \uparrow$ $\mathbb{S}\left(\lambda^{+}\right) * \mathbb{P}_{\beta}=p_{\gamma} \mid \mathbb{S}\left(\lambda^{+}\right) * \mathbb{P}_{\beta}$ for all $\beta<\gamma<\alpha$.

By $\operatorname{cf}(\alpha) \leq l^{\prime}<\lambda$, let $p_{\alpha}$ be the least upper bound of the sequence, $\left\langle p_{\beta} \mid \beta<\alpha\right\rangle$. Then, for all $j<l^{\prime}$, if $\beta=\Delta\left(g_{j}, g_{l^{\prime}}\right)$, then

$\left(\star_{j}\right) \quad p_{\alpha}\left\lceil\mathbb{S}\left(\lambda^{+}\right) * \mathbb{P}_{\beta} \geq p_{\beta}\left\lceil\mathbb{S}\left(\lambda^{+}\right) * \mathbb{P}_{\beta} \geq q_{j_{\beta} l}\left\lceil\mathbb{S}\left(\lambda^{+}\right) * \mathbb{P}_{\beta}=q_{j l}\left\lceil\mathbb{S}\left(\lambda^{+}\right) * \mathbb{P}_{\beta}\right.\right.\right.\right.$.

Put $q_{l^{\prime} l^{\prime}}:=\left(p_{\alpha}\right)^{\tau_{0}}$. Then (b) is clearly satisfied. Also, for all $j<l^{\prime}$, by $\left(\star_{j}\right)$, we get

$$
q_{l^{\prime} l^{\prime}}\left\lceil\mathbb{S}\left(\lambda^{+}\right) * \mathbb{P}_{\Delta\left(g_{j}, g_{l^{\prime}}\right)} \geq p_{\alpha}\left\lceil\mathbb{S}\left(\lambda^{+}\right) * \mathbb{P}_{\Delta\left(g_{j}, g_{l^{\prime}}\right)} \geq q_{j l}\left\lceil\mathbb{S}\left(\lambda^{+}\right) * \mathbb{P}_{\Delta\left(g_{j}, g_{l^{\prime}}\right)},\right.\right.\right.
$$

hence, we may assign $q_{j l^{\prime}}:=\operatorname{mix}\left(q_{l^{\prime} l^{\prime}}, \Delta\left(g_{j}, g_{l^{\prime}}\right), q_{j l}\right)$ for all $j<l^{\prime}$.

As $\operatorname{mix}\left(q_{l^{\prime} l^{\prime}}, \beta, q_{l^{\prime} l^{\prime}}\right)=q_{l^{\prime} l^{\prime}}$ for all $\beta<\lambda^{++}$, property (c) is satisfied, and $q_{j l} \leq q_{j l^{\prime}}$ for all $j<l^{\prime}$, which is required by clause (a). Finally, as $q_{l^{\prime} l^{\prime}} \geq p_{\alpha} \geq p_{0} \geq r_{\gamma_{0}}^{g_{l^{\prime}}}$, we conclude that all the requirements are satisfied. 
Limit step: Suppose $l^{\prime}<2^{\lambda_{0}}$ is a limit ordinal and $\left\{q_{j l} \mid j \leq l<l^{\prime}\right\}$ has already been defined. Put $\alpha:=\sup \left\{\Delta\left(g_{j}, g_{l^{\prime}}\right)+1 \mid j<l^{\prime}\right\}$, and for all $\beta<\alpha$, let $j_{\beta}$ be the least such that $j_{\beta}<l^{\prime}$ and $g_{j_{\beta}} \uparrow \beta=g_{l^{\prime}} \uparrow \beta$. By property (c) above, there exists a sequence $\left\langle s^{l} \mid l<l^{\prime}\right\rangle$ such that for every $j \leq l<l^{\prime}$, if $q_{j l}=\langle s, k\rangle$, then $s=s^{l}$. Put $\bar{s}:=\bigcup_{l<l^{\prime}} s^{l}$ and $\tau:=\operatorname{dom}(\bar{s})$.

For all $j<l^{\prime}$, let $\left\langle s_{j}, k_{j}\right\rangle$ be the least upper bound of the increasing sequence, $\left\langle q_{j l} \mid j \leq l<l^{\prime}\right\rangle$. Then by the proof of Lemma 1.5, for every $j<l^{\prime}$, either $s_{j}=\bar{s}$ or $s_{j}=\bar{s} \cup\{(\tau, 0)\}$.

- If $s_{j}=\bar{s}$ for all $j<l^{\prime}$, then let $q_{j}:=\left\langle\bar{s}, k_{j}\right\rangle$ for all $j<l^{\prime}$.

- If this is not the case, then $\operatorname{cf}(\tau)=\omega$, and for all $j<l^{\prime}$, if $\tau \in \operatorname{dom}\left(s_{j}\right)$, then $s_{j}(\tau)=0$, so let $q_{j}:=\left\langle\bar{s} \cup\{(\tau, 0)\}, k_{j}\right\rangle$ for all $j<l^{\prime}$.

Then property (a) together with the above choice implies:

$\left(\mathrm{a}^{\prime}\right) r_{\gamma_{0}}^{g_{j_{\beta}}} \leq q_{j_{\beta} j_{\beta}} \leq\left\langle s_{j_{\beta}}, k_{j_{\beta}}\right\rangle \leq q_{j_{\beta}}$ for all $\beta<\alpha$

$\left(\mathrm{c}^{\prime}\right) q_{j_{\beta}}\left|\mathbb{S}\left(\lambda^{+}\right) * \mathbb{P}_{\beta}=q_{j_{\gamma}}\right| \mathbb{S}\left(\lambda^{+}\right) * \mathbb{P}_{\beta}$ for all $\beta<\gamma<\alpha$.

Thus, to compare with the successor step, we now work against $\left\{q_{j} \mid j<l^{\prime}\right\}$ instead of $\left\{q_{j l} \mid j<l^{\prime}\right\}$, where $l$ was the immediate predecessor of $l^{\prime}$.

For all $\beta<\alpha$, let $p_{\beta}:=\operatorname{mix}\left(q_{j_{\beta}}, \beta, r_{\gamma_{0}}^{g_{l^{\prime}}}\right)$, and let $p_{\alpha}$ be the least upper bound of the increasing sequence $\left\langle p_{\beta} \mid \beta<\alpha\right\rangle$. Then, for all $j<l^{\prime}$, if $\beta=\Delta\left(g_{j}, g_{l^{\prime}}\right)$, then

$\left(\star_{j}^{\prime}\right) \quad p_{\alpha}\left\lceil\mathbb{S}\left(\lambda^{+}\right) * \mathbb{P}_{\beta} \geq p_{\beta}\left\lceil\mathbb{S}\left(\lambda^{+}\right) * \mathbb{P}_{\beta} \geq q_{j_{\beta}}\left\lceil\mathbb{S}\left(\lambda^{+}\right) * \mathbb{P}_{\beta}=q_{j}\left\lceil\mathbb{S}\left(\lambda^{+}\right) * \mathbb{P}_{\beta}\right.\right.\right.\right.$.

Thus, put $q_{l^{\prime} l^{\prime}}:=\left(p_{\alpha}\right)^{\tau_{0}}$, and for all $j<l^{\prime}$, let $q_{j l^{\prime}}:=\operatorname{mix}\left(q_{l^{\prime} l^{\prime}}, \Delta\left(g_{j}, g_{l^{\prime}}\right), q_{j}\right)$.

This completes the construction of $\mathcal{F}_{0}=\left\{q_{g} \mid g \in{ }^{Z_{0}} 2\right\}$.

Next, suppose $\left\langle\left(\gamma_{m}, Y_{m}, Z_{m}, \mathcal{F}_{m}\right) \mid m \leq n\right\rangle$ has already been defined for some $n<\omega$. Let:

- $\gamma_{n+1}:=\sup \left\{\max \left(c_{i}^{k}\right) \mid\langle s, k\rangle \in \mathcal{F}_{n}\right.$ for some s, $\left.i \in \operatorname{supp}(k)\right\}$;

- $Y_{n+1}:=\bigcup\left\{\operatorname{supp}(k) \mid\langle s, k\rangle \in \mathcal{F}_{n}\right.$ for some $\left.s\right\}$

- $Z_{n+1}:=\bigcup\left\{\psi_{Y_{k}}\right.$ " $\left.\lambda_{n+1} \mid k \leq n+1\right\}$;

- $\mathcal{F}_{n+1}:=\left\{q_{g} \mid g \in Z^{Z_{n+1}} 2\right\}$.

Clearly, our main task is defining $q_{g}$ for all $g \in Z^{Z_{n+1}} 2$. Let $\left\{g_{j} \mid j<2^{\lambda_{n+1}}\right\}$ be the $<_{\chi}$-least bijective enumeration of $Z_{n+1} 2$. We shall now define an upper triangular matrix $\left\{q_{j l} \mid j \leq l<2^{\lambda_{n+1}}\right\}$ in such a way that for all $j \leq j^{\prime} \leq l \leq l^{\prime}<2^{\lambda_{n+1}}$, the following holds:

(a) $\left(q_{g_{j} \backslash Z_{n}}\right)_{\gamma_{n+1}}^{g_{j} \backslash\left(Z_{n+1} \backslash Z_{n}\right)} \leq q_{j j} \leq q_{j l} \leq q_{j l^{\prime}}$

(b) $p^{\tau_{n+1}} \leq q_{j j}$ for some condition $p \in \mathbb{Q}^{\prime}\left(\lambda^{+}\right)$;

(c) $q_{j l^{\prime}} \mid \mathbb{S}\left(\lambda^{+}\right) * \mathbb{P}_{\beta}=q_{j^{\prime} l^{\prime}}\left\lceil\mathbb{S}\left(\lambda^{+}\right) * \mathbb{P}_{\beta}\right.$ whenever $g_{j}\left\lceil\beta=g_{j^{\prime}} \uparrow \beta\right.$.

As in the base case, once the matrix is defined, for each $g \in Z_{n+1} 2$, we pick the unique $j$ such that $g_{j}=g$, and let $q_{g}$ be the least upper bound of the increasing sequence $\left\langle q_{j l} \mid j \leq l<2^{\lambda_{n+1}}\right\rangle$.

Induction base: Let $q_{00}:=\left(\left(q_{g_{0}} \backslash Z_{n}\right)_{\gamma_{n+1}}^{g_{0}\left\lceil\left(Z_{n+1} \backslash Z_{n}\right)\right.}\right)^{\tau_{n+1}}$.

Successor step: Suppose $l<2^{\lambda_{n+1}}$, and $\left\{q_{j l} \mid j \leq l\right\}$ has already been defined. We would like to define $\left\{q_{j l^{\prime}} \mid j \leq l^{\prime}\right\}$, for $l^{\prime}:=l+1$.

Put $\alpha:=\sup \left\{\Delta\left(g_{j}, g_{l^{\prime}}\right)+1 \mid j<l^{\prime}\right\}$, and for all $\beta<\alpha$, let $j_{\beta}$ be the least such that $j_{\beta}<l^{\prime}$ and $g_{j_{\beta}}\left\lceil\beta=g_{l^{\prime}} \uparrow \beta\right.$. Fix $\beta<\alpha$. By (5), we have $q_{g_{l^{\prime}}}\left|Z_{n}\right| \mathbb{S}\left(\lambda^{+}\right) * \mathbb{P}_{\beta}=$ $q_{g_{j_{\beta}} \backslash Z_{n}}\left\lceil\mathbb{S}\left(\lambda^{+}\right) * \mathbb{P}_{\beta}\right.$, and hence

$$
\left(q_{g^{\prime}} \uparrow Z_{n}\right)_{\gamma_{n+1}}^{g_{l^{\prime}} \uparrow\left(Z_{n+1} \backslash Z_{n}\right)} \uparrow \mathbb{S}\left(\lambda^{+}\right) * \mathbb{P}_{\beta}=\left(q_{g_{\beta}} \uparrow Z_{n}\right)_{\gamma_{n+1}}^{g_{j_{\beta}} \uparrow\left(Z_{n+1} \backslash Z_{n}\right)} \uparrow \mathbb{S}\left(\lambda^{+}\right) * \mathbb{P}_{\beta} .
$$


In addition, by property (a), we have $\left(q_{g_{j_{\beta}} \backslash Z_{n}}\right)_{\gamma_{n+1}}^{g_{j_{\beta}} \uparrow\left(Z_{n+1} \backslash Z_{n}\right)} \leq q_{j_{\beta} l}$. Denote $r_{l^{\prime}}:=$ $\left(q_{l^{\prime}} \backslash Z_{n}\right)_{\gamma_{n+1}}^{g_{l^{\prime}} \uparrow\left(Z_{n+1} \backslash Z_{n}\right)}$. It follows that $r_{l^{\prime}} \uparrow \mathbb{S}\left(\lambda^{+}\right) * \mathbb{P}_{\beta} \leq q_{j_{\beta} l} \uparrow \mathbb{S}\left(\lambda^{+}\right) * \mathbb{P}_{\beta}$, and hence $\operatorname{mix}\left(q_{j_{\beta}}, \beta, r_{l^{\prime}}\right)$ is a well-defined extension of $r_{l^{\prime}}$.

By property (c), we get that $\beta<\gamma<\alpha$ implies $q_{j_{\beta} l}\left\lceil\mathbb{S}\left(\lambda^{+}\right) * \mathbb{P}_{\beta}=q_{j_{\gamma} l} \uparrow\right.$ $\mathbb{S}\left(\lambda^{+}\right) * \mathbb{P}_{\beta}$ and $\operatorname{mix}\left(q_{j_{\beta} l}, \beta, r_{l^{\prime}}\right)=\operatorname{mix}\left(q_{j_{\gamma} l}, \beta, r_{l^{\prime}}\right) \leq \operatorname{mix}\left(q_{j_{\gamma} l}, \gamma, r_{l^{\prime}}\right)$.

For all $\beta<\alpha$, let $p_{\beta}:=\operatorname{mix}\left(q_{j_{\beta}}, \beta, r_{l^{\prime}}\right)$. Then, we have just established that $\left\langle p_{\beta} \mid \beta<\alpha\right\rangle$ is an increasing sequence of conditions, with $p_{0} \geq\left(q_{g_{l^{\prime}} \uparrow Z_{n}}\right)_{\gamma_{n+1}}^{g_{l^{\prime}} \uparrow\left(Z_{n+1} \backslash Z_{n}\right)}$, and $p_{\beta}\left\lceil\mathbb{S}\left(\lambda^{+}\right) * \mathbb{P}_{\beta}=p_{\gamma}\left\lceil\mathbb{S}\left(\lambda^{+}\right) * \mathbb{P}_{\beta}\right.\right.$ for all $\beta<\gamma<\alpha$. By $\operatorname{cf}(\alpha)<\lambda$, let $p_{\alpha}$ be the least upper bound of the sequence, $\left\langle p_{\beta} \mid \beta<\alpha\right\rangle$. Then, for all $j<l^{\prime}$, we have $\left(\star_{j}\right)$ as above, so let $q_{l^{\prime} l^{\prime}}:=\left(p_{\alpha}\right)^{\tau_{n+1}}$ and assign $q_{j l^{\prime}}:=\operatorname{mix}\left(q_{l^{\prime} l^{\prime}}, \Delta\left(g_{j}, g_{l^{\prime}}\right), q_{j l}\right)$ for all $j<l^{\prime}$.

Limit step: Suppose $l^{\prime}<2^{\lambda_{n+1}}$ is some ordinal and $\left\{q_{j l} \mid j \leq l<l^{\prime}\right\}$ has already been defined. Put $\alpha:=\sup \left\{\Delta\left(g_{j}, g_{l^{\prime}}\right)+1 \mid j<l^{\prime}\right\}$ and, for all $\beta<\alpha$, let $j_{\beta}$ be the least such that $j_{\beta}<l^{\prime}$ and $g_{j_{\beta}} \uparrow \beta=g_{l^{\prime}} \uparrow \beta$. By property (c) above, there exists a sequence $\left\langle s^{l} \mid l<l^{\prime}\right\rangle$ such that for every $j \leq l<l^{\prime}$, if $q_{j l}=\langle s, k\rangle$, then $s=s^{l}$. Put $\bar{s}:=\bigcup_{l<l^{\prime}} s^{l}$ and $\tau:=\operatorname{dom}(\bar{s})$.

For all $j<l^{\prime}$, let $\left\langle s_{j}, k_{j}\right\rangle$ be the least upper bound of the increasing sequence, $\left\langle q_{j l} \mid j \leq l<l^{\prime}\right\rangle$. Then for every $j<l^{\prime}$, either $s_{j}=\bar{s}$ or $s_{j}=\bar{s} \cup\{(\tau, 0)\}$.

- If $s_{j}=\bar{s}$ for all $j<l^{\prime}$, then let $q_{j}:=\left\langle\bar{s}, k_{j}\right\rangle$ for all $j<l^{\prime}$.

- If this is not the case, then let $q_{j}:=\left\langle\bar{s} \cup\{(\tau, 0)\}, k_{j}\right\rangle$ for all $j<l^{\prime}$.

Then, the following holds:

(a') $\left(q_{g_{j_{\beta}}} \backslash Z_{n}\right)_{\gamma_{n+1}}^{g_{j_{\beta}} \backslash\left(Z_{n+1} \backslash Z_{n}\right)} \leq q_{j_{\beta} j_{\beta}} \leq q_{j_{\beta}}$ for all $\beta<\alpha$;

$\left(\mathrm{c}^{\prime}\right) q_{j_{\beta}}\left\lceil\mathbb{S}\left(\lambda^{+}\right) * \mathbb{P}_{\beta}=q_{j_{\gamma}} \mid \mathbb{S}\left(\lambda^{+}\right) * \mathbb{P}_{\beta}\right.$ for all $\beta<\gamma<\alpha$.

Fix $\beta<\alpha$. By (5), we have $q_{g_{l^{\prime}}} \mid Z_{n}\left\lceil\mathbb{S}\left(\lambda^{+}\right) * \mathbb{P}_{\beta}=q_{g_{j_{\beta}}} \mid Z_{n}\left\lceil\mathbb{S}\left(\lambda^{+}\right) * \mathbb{P}_{\beta}\right.\right.$, and hence

$$
\left(q_{g_{l^{\prime}} \uparrow Z_{n}}\right)_{\gamma_{n+1}}^{g_{\gamma^{\prime}} \uparrow\left(Z_{n+1} \backslash Z_{n}\right)}\left\lceil\mathbb{S}\left(\lambda^{+}\right) * \mathbb{P}_{\beta}=\left(q_{g_{\beta}} \backslash Z_{n}\right)_{\gamma_{n+1}}^{g_{j_{\beta}} \uparrow\left(Z_{n+1} \backslash Z_{n}\right)}\left\lceil\mathbb{S}\left(\lambda^{+}\right) * \mathbb{P}_{\beta} .\right.\right.
$$

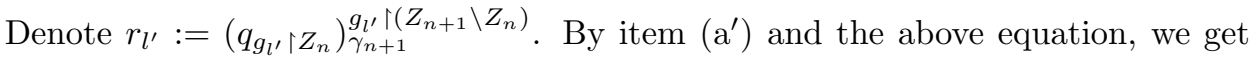
that $r_{l^{\prime}}\left\lceil\mathbb{S}\left(\lambda^{+}\right) * \mathbb{P}_{\beta} \leq q_{j_{\beta}}\left\lceil\mathbb{S}\left(\lambda^{+}\right) * \mathbb{P}_{\beta}\right.\right.$, and hence $\operatorname{mix}\left(q_{j_{\beta}}, \beta, r_{l^{\prime}}\right)$ is a well-defined extension of $r_{l^{\prime}}$. By $\left(\mathrm{c}^{\prime}\right)$, if we write $p_{\beta}:=\operatorname{mix}\left(q_{j_{\beta}}, \beta, r_{l^{\prime}}\right)$, then $\left\langle p_{\beta} \mid \beta<\alpha\right\rangle$ is an increasing sequence of conditions, with $p_{0} \geq\left(q_{g_{l^{\prime} \backslash Z_{n}}}\right)_{\gamma_{n+1}}^{g_{l^{\prime}} \backslash\left(Z_{n+1} \backslash Z_{n}\right)}$, and $p_{\beta} \uparrow$ $\mathbb{S}\left(\lambda^{+}\right) * \mathbb{P}_{\beta}=p_{\gamma} \mid \mathbb{S}\left(\lambda^{+}\right) * \mathbb{P}_{\beta}$ for all $\beta<\gamma<\alpha$.

Let $p_{\alpha}$ be the least upper bound of the sequence, $\left\langle p_{\beta} \mid \beta<\alpha\right\rangle$. Then, for all $j<$ $l^{\prime}$, we have $\left(\star_{j}^{\prime}\right)$ as above, so let $q_{l^{\prime} l^{\prime}}:=\left(p_{\alpha}\right)^{\tau_{n+1}}$ and $q_{j l^{\prime}}:=\operatorname{mix}\left(q_{l^{\prime} l^{\prime}}, \Delta\left(g_{j}, g_{l^{\prime}}\right), q_{j}\right)$ for all $j<l^{\prime}$.

This completes the construction of $\mathcal{F}_{n+1}=\left\{q_{g} \mid g \in{ }^{Z_{n+1}} 2\right\}$.

Claim 1.8.5. $\left\{\gamma_{n}, Z_{n}, Y_{n}, \mathcal{F}_{n}\right\} \subseteq M$ for all $n<\omega$.

Proof. Easy.

Put $Z:=\bigcup_{n<\omega} Z_{n}$. We now state and prove several claims that should gradually clarify the role of the above construction.

Claim 1.8.6. For every $g \in{ }^{Z} 2$, if $\langle s, k\rangle$ is the least upper bound of the increasing sequence $\left\langle q_{g \mid Z_{n}} \mid n<\omega\right\rangle$, then $\operatorname{supp}(k)=Z$.

Proof. Fix $n<\omega$ and $\left\langle s_{n}, k_{n}\right\rangle \in \mathcal{F}_{n}$. By property (4) and Definition 1.8.2, we have $Z_{n} \backslash Z_{n-1} \subseteq \operatorname{supp}\left(k_{n}\right)$. By definition of $Y_{n+1}$, we also have $\operatorname{supp}\left(k_{n}\right) \subseteq Y_{n+1}$. 
It follows that if $\langle s, k\rangle$ is the least upper bound of an increasing sequence $\left\langle\left\langle s_{n}, k_{n}\right\rangle\right|$ $n<\omega\rangle \in \prod_{n<\omega} \mathcal{F}_{n}$, then

$$
Z=\bigcup_{n<\omega}\left(Z_{n} \backslash Z_{n-1}\right) \subseteq \operatorname{supp}(k) \subseteq \bigcup_{n<\omega} Y_{n+1}=Z .
$$

Definition 1.8.7. For every $\beta<\lambda^{++}, h: Z \cap \beta \rightarrow 2$, and $i \in Z \cap \beta$, we define a pair $\left(x_{i}^{\stackrel{h}{h}}, c_{i}^{\stackrel{h}{h}}\right)$.

Pick a function $g: Z \rightarrow 2$ that extends $h$. For each $n<\omega$, denote $\left\langle s_{n}, k_{n}\right\rangle:=$ $q_{g \nmid Z_{n}}$, and then let

$$
\begin{gathered}
x_{i}^{h}:=\bigcup\left\{x_{i}^{k_{n}} \mid n<\omega \& i \in \operatorname{supp}\left(k_{n}\right)\right\}, \\
c_{i}^{h}:=\operatorname{cl}\left(\bigcup\left\{c_{i}^{k_{n}} \mid n<\omega \& i \in \operatorname{supp}\left(k_{n}\right)\right\}\right) .
\end{gathered}
$$

Notice that by property (5) of the construction, the above definition does not depend on the choice of the function $g$.

Claim 1.8.8. For all $\beta<\lambda^{++}, h: Z \cap \beta \rightarrow 2$, and $i \in Z \cap \beta$, we have

$$
\sup \left(x_{i}^{h}\right)=\max \left(c_{i}^{h}\right)=\tau .
$$

Proof. Pick $g: Z \rightarrow 2$ extending $h$. Fix $n<\omega$, and denote $\left\langle s_{n}, k_{n}\right\rangle=q_{g \mid Z_{n}}$. By property (6) of the construction, we have $\operatorname{dom}\left(s_{n}\right)>\tau_{n}$. By $\left\langle s_{n}, k_{n}\right\rangle \in \mathbb{Q}^{\prime}\left(\lambda^{+}\right) \cap M$, we have

$$
\tau=\lambda^{+} \cap M>\sup \left(x_{i}^{k_{n}}\right)=\max \left(c_{i}^{k_{n}}\right)=\sup (\operatorname{dom}(s)) \geq \tau_{n} .
$$

The conclusion now follows.

Claim 1.8.9. There exists $\bar{s}: \tau \rightarrow 2$ such that for all $g \in{ }^{Z}$, if $\langle s, k\rangle$ is the least upper bound of the increasing sequence $\left\langle q_{g \mid Z_{n}} \mid n<\omega\right\rangle$, then $s \in\{\bar{s}, \bar{s} \cup\{(\tau, 0)\}\}$.

Proof. By property (5) of the construction, there exists a sequence $\left\langle s^{n} \mid n<\omega\right\rangle$ such that for every $n<\omega$ and $\langle s, k\rangle \in \mathcal{F}_{n}$, we have $s^{n}=s$. Put $\bar{s}:=\bigcup_{n<\omega} s^{n}$.

If $\left\langle s^{\prime}, k^{\prime}\right\rangle \in \mathcal{F}_{n}$, then $s^{\prime} \in M$, and hence $\operatorname{dom}\left(s^{\prime}\right) \subseteq \tau=M \cap \lambda^{+}$. In particular, $\operatorname{dom}(\bar{s}) \subseteq \tau$.

Finally, for every function $g \in{ }^{Z}$, the increasing sequence $\left\langle q_{g \mid Z_{n}} \mid n<\omega\right\rangle$ is an element of $\prod_{n<\omega} \mathcal{F}_{n}$, so by the proof of Lemma 1.5] if $\langle s, k\rangle$ is the least upper bound of the above sequence, then either $s=\bar{s}$ or $s=\bar{s} \cup\{(\tau, 0)\}$.

Claim 1.8.10. For every $g \in{ }^{Z}$, if $q$ is an upper bound of the increasing sequence $\left\langle q_{g \mid Z_{n}} \mid n<\omega\right\rangle$, then $q \Vdash \check{\tau} \in \dot{E}$.

Proof. Fix $g$ and $q$ as above. For all $n<\omega$, by property (6) and $\mathcal{F}_{n} \subseteq M$, there exists $\alpha_{n}$ with $\tau_{n} \leq \alpha_{n}<\lambda^{+} \cap M=\tau$ such that $q_{g \mid Z_{n}} \Vdash \check{\alpha_{n}} \in \dot{E}$. Since $\sup \left\{\tau_{n} \mid n<\omega\right\}=\tau$, we get that $\sup \left\{\alpha_{n} \mid n<\omega\right\}=\tau$, and hence $q$ forces that $\tau$ is an accumulation point of the club $E$.

Thus, our main task is to argue the existence of some $g \in{ }^{Z} 2$ for which there exists an upper bound of the increasing sequence $\left\langle q_{g \mid Z_{n}}\right| n\langle\omega\rangle$ which is compatible with $\langle\bar{s} \cup\{(\tau, 1)\}, \emptyset\rangle$. Evidently, such a condition will force that $S$ meets $E$. A key fact for ensuring the existence of such a function is the next claim. 
Claim 1.8.11. Suppose $A \subseteq \tau$.

For every $i \in Z$, there exists some $m<2$ such that $x_{i}^{h} \neq A$ for every function $h: Z \cap \beta \rightarrow 2$ with $i<\beta<\lambda^{++}$and $h(i)=m$.

Proof. Suppose $i \in Z$. Let $n<\omega$ be the unique ordinal such that $i \in Z_{n} \backslash Z_{n-1}$. Since $\left(\left(\gamma_{n}+0, \gamma_{n}+\omega\right)\right) \neq\left(\left(\gamma_{n}+1, \gamma_{n}+\omega\right)\right)$ and $\gamma_{n}+\omega<\tau=M \cap \lambda^{+}$, let us pick some $m<2$ such that $\left(\left(\gamma_{n}+m, \gamma_{n}+\omega\right)\right) \neq A \cap\left(\left(\gamma_{n}, \gamma_{n}+\omega\right)\right)$.

To see that $m$ works, suppose that $h$ is as in the hypothesis and that $g \in{ }_{2}$ extends $h$. In particular, $g(i)=m$. Put $\left\langle s_{n}, k_{n}\right\rangle:=q_{g \mid Z_{n}}$. Then by property (4) of the construction, $\left\langle s_{n}, k_{n}\right\rangle \geq r_{\gamma_{n}}^{g \uparrow\left(Z_{n} \backslash Z_{n-1}\right)} \geq r$ for some condition $r$, and so by Definition 1.8.2, this means that $i \in \operatorname{supp}(k)$ and

$x_{i}^{k_{n}} \cap\left(\left(\gamma_{n}, \gamma_{n}+\omega\right)\right)=\left(\left(\gamma_{n}+g(i), \gamma_{n}+\omega\right)\right)=\left(\left(\gamma_{n}+m, \gamma_{n}+\omega\right)\right) \neq A \cap((\gamma, \gamma+\omega))$.

In particular $x_{i}^{k_{n}} \cap \sup \left(x_{i}^{k_{n}}\right) \neq A \cap \sup \left(x_{i}^{k_{n}}\right)$. As $x_{i}^{k_{n}} \sqsubseteq x_{i}^{h}$, we conclude that $x_{i}^{\stackrel{h}{h}} \neq A$.

Let $D$ denote the set of all $\langle s, k\rangle$ such that all items of Definition 1.4, except for (5), are satisfied, and where instead we require that for all $\alpha \in \operatorname{supp}(k)$ :

(5a) either $\sup \left(x_{\alpha}^{k}\right)=\max \left(c_{\alpha}^{k}\right)=\sup (\operatorname{dom}(s))$ or

(5b) $\alpha \in \operatorname{supp}\left(k^{*}\right)$ and $\left(x_{\alpha}^{k}, c_{\alpha}^{k}\right)=\left(x_{\alpha}^{k^{*}}, c_{\alpha}^{k^{*}}\right)$.

Then $D \supseteq \mathbb{Q}^{\prime}\left(\lambda^{+}\right)$is a dense set and, by a proof similar to the one of Lemma 1.5. every increasing sequence of length $<\lambda^{+}$of elements of $D$ has a minimal upper bound within $D$.

For each $\alpha<\lambda^{++}$, let $D_{\alpha}$ denote the dense open subset of $\mathbb{S}\left(\lambda^{+}\right) * \mathbb{P}_{\alpha}$ that decides $\overrightarrow{A_{\alpha}}(\tau)$, and let $D_{\alpha}^{\rightarrow}:=\left\{p \in D|p| \mathbb{S}\left(\lambda^{+}\right) * \mathbb{P}_{\alpha} \in D_{\alpha}\right\}$ denote its cylindric extension within $D$.

Put $\theta:=\operatorname{otp}(Z)$ and let $\left\{\varepsilon_{\alpha} \mid \alpha<\theta\right\}$ be the increasing enumeration of $Z$. We now define by induction an increasing sequence of conditions $\left\langle p_{\alpha}=\left\langle s_{\alpha}, k_{\alpha}\right\rangle \mid \alpha<\theta\right\rangle$ and a chain of functions $\left\{h_{\alpha}: Z \cap\left(\varepsilon_{\alpha}+1\right) \rightarrow 2 \mid \alpha<\theta\right\}$ in such a way that for all $\alpha<\theta$ :

(a) $\left\langle\bar{s} \cup\{(\tau, 1)\}, k^{*}\right\rangle \leq\left\langle s_{\alpha}, k_{\alpha}\right\rangle \in D_{\varepsilon_{\alpha}}^{\rightarrow}$;

(b) $Z \cap\left(\varepsilon_{\alpha}+1\right) \subseteq \operatorname{supp}\left(k_{\alpha}\right) \subseteq \operatorname{supp}\left(k^{*}\right) \cup\left(\varepsilon_{\alpha}+1\right)$;

(c) $x_{i}^{h_{\alpha}} \sqsubseteq x_{i}^{k_{\alpha}}$ and $c_{i}^{h_{\alpha}} \sqsubseteq c_{i}^{k_{\alpha}}$ for all $i \in Z \cap\left(\varepsilon_{\alpha}+1\right)$;

(d) $\left(x_{i}^{k_{\alpha}}, c_{i}^{k_{\alpha}}\right)=\left(x_{i}^{k^{*}}, c_{i}^{k^{*}}\right)$ for all $i \in \operatorname{supp}\left(k_{\alpha}\right) \backslash\left(\varepsilon_{\alpha}+1\right)$.

Induction base: Since $0 \in \operatorname{supp}\left(k^{*}\right) \subseteq Z$, we have $\varepsilon_{0}=0$. Pick $\left\langle s_{0}, \emptyset\right\rangle \in D_{0}$ with $\bar{s} \cup\{(\tau, 1)\} \sqsubseteq s_{0} 5$ In particular, $\left\langle s_{0}, \emptyset\right\rangle$ decides $\overrightarrow{A_{0}}(\tau)$ to be, say, $A_{0}^{\tau}$. Since $D_{0}$ is a dense open set, we may moreover assume that $\operatorname{dom}\left(s_{0}\right)$ is the successor of a limit ordinal $\gamma$ and that $s_{0}(\gamma)=0$.

By Claim 1.8.11, pick a function $h_{0}:\left\{\varepsilon_{0}\right\} \rightarrow 2$ such that $A_{0}^{\tau} \neq x_{\varepsilon_{0}}^{h_{0}}$, and let $p_{0}:=\left\langle s_{0}, k_{0}\right\rangle$ be the condition in $D$ such that $\operatorname{supp}\left(k_{0}\right)=\operatorname{supp}\left(k^{*}\right)$ and for all $i \in \operatorname{supp}\left(k_{0}\right)$ :

$$
\left(x_{i}^{k_{0}}, c_{i}^{k_{0}}\right):= \begin{cases}\left(x_{i}^{h_{0}} \cup((\tau, \gamma)), c_{i}^{h_{0}} \cup\{\gamma\}\right), & i=\varepsilon_{0}, \\ \left(x_{i}^{k^{*}}, c_{i}^{k^{*}}\right), & \text { else. }\end{cases}
$$

\footnotetext{
${ }^{5}$ Recall that $D_{0} \subseteq \mathbb{S}\left(\lambda^{+}\right) * \mathbb{P}_{0}$ and that $\mathbb{P}_{0}$ is the trivial forcing $\langle\{\emptyset\},\{(\emptyset, \emptyset)\}\rangle$
} 
Let us show that $p_{0}$ is indeed a well-defined condition. Assume towards a contradiction that this is not the case. Hence, we can fix $i \in \operatorname{supp}\left(k_{0}\right), t \geq s_{0}$ and $\delta \in \underline{t} \cap c_{i}^{k_{0}}$ such that $\overrightarrow{A_{i}}(\delta)=x_{i}^{k_{0}} \cap \delta$. Clearly, if $i>\varepsilon_{0}$, then $\delta \in \underline{t} \cap c_{i}^{k^{*}}$ implies that $\delta \in \underline{s^{*}}$, contradicting the fact that $\left\langle s^{*}, k^{*}\right\rangle$ is a legitimate condition. So $i=\varepsilon_{0}=0$.

Since $\delta \in c_{0}^{h_{0}}$, we get from Claim 1.8.8 that either $\delta \in\{\tau, \gamma\}$ or $\delta \in \underline{\bar{s}}$. It is impossible that $\delta=\tau$, because the choice of $h_{0}$ ensured that $x_{0}^{k_{0}} \cap \delta \neq \overrightarrow{A_{0}}(\delta)$. It is also impossible that $\delta=\gamma$, because $t \geq s_{0}$ and $s_{0}(\gamma)=0$. So, $\delta \in \underline{\bar{s}}$.

Pick a function $g: Z \rightarrow 2$ extending $h_{0}$, and let $\left\langle s^{n}, k^{n}\right\rangle:=q_{g \mid Z_{n}}$ for all $n<$ $\omega$. Then $\bar{s}=\bigcup_{n<\omega} s^{n}, x_{0}^{h_{0}}=\bigcup_{n<\omega} x_{0}^{k^{n}}$ and $c_{0}^{h_{0}}=\bigcup_{n<\omega} c_{0}^{k^{n}} \cup\{\tau\}$. Pick $n<$ $\omega$ such that $\delta \in \underline{s_{n}}$. By $\left\langle s^{n}, k^{n}\right\rangle \in \mathbb{Q}^{\prime}\left(\lambda^{+}\right)$, we have $\sup \left(x_{0}^{k^{n}}\right)=\max \left(x_{0}^{k^{n}}\right)=$ $\sup \left(\operatorname{dom}\left(s^{n}\right)\right) \geq \delta$, and then $\overrightarrow{A_{0}}(\delta)=x_{0}^{h_{0}} \cap \delta=x_{0}^{k^{n}} \cap \delta$ while $\delta \in \underline{s^{n}} \cap c_{0}^{k^{n}}$, contradicting the basic fact that $\left\langle s^{n}, k^{n}\right\rangle$ is a legitimate condition.

Thus, $p_{0}$ is a well-defined element of $D_{\varepsilon_{0}}$ and satisfies requirements (a)-(d).

Successor step: Suppose that for some $\alpha<\theta, p_{\alpha}$ has already been defined. Pick $p^{\prime}=\left\langle s^{\prime}, k^{\prime}\right\rangle \geq p_{\alpha}$ in $D_{\varepsilon_{\alpha+1}}^{\rightarrow}$ with $\operatorname{dom}\left(s^{\prime}\right)=\gamma+1$ and $s^{\prime}(\gamma)=0$ for some limit $\gamma<\lambda^{+}$. Then $p^{\prime} \mid \mathbb{S}\left(\lambda^{+}\right) * \mathbb{P}_{\varepsilon_{\alpha+1}}$ decides $\overrightarrow{A_{\varepsilon_{\alpha+1}}}(\tau)$ to be, say, $A_{\varepsilon_{\alpha+1}}^{\tau}$. By Claim 1.8.11, pick a function $h_{\alpha+1}: Z \cap\left(\varepsilon_{\alpha+1}+1\right) \rightarrow 2$ extending $h_{\alpha}$ such that $A_{\varepsilon_{\alpha+1}}^{\tau} \neq x_{\varepsilon_{\alpha+1}}^{h_{\alpha+1}}$. Finally, let $p_{\alpha+1}=\left\langle s_{\alpha+1}, k_{\alpha+1}\right\rangle$ be the condition in $D$ such that:

- $s_{\alpha+1}=s^{\prime}$;

- $\operatorname{supp}\left(k_{\alpha+1}\right)=\left(\operatorname{supp}\left(k^{\prime}\right) \cap \varepsilon_{\alpha+1}\right) \cup\left\{\varepsilon_{\alpha+1}\right\} \cup \operatorname{supp}\left(k^{*}\right)$;

- for all $i \in \operatorname{supp}\left(k_{\alpha+1}\right)$ :

$$
\left(x_{i}^{k_{\alpha+1}}, c_{i}^{k_{\alpha+1}}\right):= \begin{cases}\left(x_{i}^{k^{\prime}}, c_{i}^{k^{\prime}}\right), & i<\varepsilon_{\alpha+1}, \\ \left(x_{i}^{h_{\alpha+1}} \cup((\tau, \gamma)), c_{i}^{h_{\alpha+1}} \cup\{\gamma\}\right), & i=\varepsilon_{\alpha+1}, \\ \left(x_{i}^{k^{*}}, c_{i}^{k^{*}}\right), & \text { else. }\end{cases}
$$

Assume towards a contradiction that $p_{\alpha+1}$ is not a well-defined condition. Fix $i \in \operatorname{supp}\left(k_{\alpha+1}\right)$ and $\delta \in \underline{s_{\alpha+1}} \cap c_{i}^{k_{\alpha+1}}$ such that $\overrightarrow{A_{i}}(\delta)=x_{i}^{k_{\alpha+1}} \cap \delta$. If $i \neq \varepsilon_{\alpha+1}$, then we get a contradiction to the fact that $\left\langle s^{*}, k^{*}\right\rangle$ and $\left\langle s^{\prime}, k^{\prime}\right\rangle$ are legitimate conditions. If $i=\varepsilon_{\alpha+1}$, then Claim 1.8 .8 and the choice of the function $h_{\alpha+1}$ ensures that $\delta \in s_{\alpha+1} \cap \tau$. But then, $\delta \in \underline{\bar{s}}$, and so by the exact same argument as the induction base, we have $\overrightarrow{A_{\varepsilon_{\alpha+1}}}(\delta) \neq x_{\varepsilon_{\alpha+1}}^{k_{\alpha+1}} \cap \delta$.

Clearly, $p_{\alpha+1}$ satisfies requirements (a)-(d).

Limit step: Suppose that $\left\langle p_{\beta} \mid \beta<\alpha\right\rangle$ and $\left\{h_{\beta}: Z \cap\left(\varepsilon_{\beta}+1\right) \rightarrow 2 \mid \beta<\alpha\right\}$ has already been defined for some limit ordinal $\alpha<\theta$.

Since $\alpha<\theta<\lambda^{+}$, let $\langle s, k\rangle$ be the least upper bound of $\left\langle p_{\beta} \mid \beta<\alpha\right\rangle$. Note that $\left(x_{i}^{k}, c_{i}^{k}\right)=\left(x_{i}^{k^{*}}, c_{i}^{k^{*}}\right)$ for all $i \in \operatorname{supp}(k) \backslash \varepsilon_{\alpha}$. Fix $\left\langle s^{\prime}, k^{\prime}\right\rangle \geq\langle s, k\rangle$ in $D_{\varepsilon_{\alpha}}^{\rightarrow}$ with $\operatorname{dom}\left(s^{\prime}\right)=\gamma+1$ and $s^{\prime}(\gamma)=0$ for some limit $\gamma\left\langle\lambda^{+}\right.$. Then $\left\langle s^{\prime}, k^{\prime}\right\rangle\left\lceil\mathbb{S}\left(\lambda^{+}\right) * \mathbb{P}_{\varepsilon_{\alpha}}\right.$ decides $\overrightarrow{A_{\varepsilon_{\alpha}}}(\tau)$ to be, say, $A_{\varepsilon_{\alpha}}^{\tau}$. Put $h:=\bigcup_{\beta<\alpha} h_{\beta}$ and pick $h_{\alpha}: Z \cap\left(\varepsilon_{\alpha}+1\right) \rightarrow 2$ extending $h$ such that $A_{\varepsilon_{\alpha}}^{\tau} \neq x_{\varepsilon_{\alpha}}^{h_{\alpha}}$. Finally, let $p_{\alpha}=\left\langle s_{\alpha}, k_{\alpha}\right\rangle$ be the condition in $D$ such that:

- $s_{\alpha}=s^{\prime}$

- $\operatorname{supp}\left(k_{\alpha}\right)=\left(\operatorname{supp}\left(k^{\prime}\right) \cap \varepsilon_{\alpha}\right) \cup\left\{\varepsilon_{\alpha}\right\} \cup \operatorname{supp}\left(k^{*}\right)$; 
- for all $i \in \operatorname{supp}\left(k_{\alpha}\right)$ :

$$
\left(x_{i}^{k_{\alpha}}, c_{i}^{k_{\alpha}}\right):= \begin{cases}\left(x_{i}^{k^{\prime}}, c_{i}^{k^{\prime}}\right), & i<\varepsilon_{\alpha}, \\ \left(x_{i}^{h_{\alpha}} \cup((\tau, \gamma)), c_{i}^{h_{\alpha}} \cup\{\gamma\}\right), & i=\varepsilon_{\alpha}, \\ \left(x_{i}^{k^{*}}, c_{i}^{k^{*}}\right), & \text { else. }\end{cases}
$$

Then $p_{\alpha}$ is a well-defined condition that satisfies all the requirements.

This completes the construction. Put $g:=\bigcup_{\alpha<\theta} h_{\alpha}$, and let $p$ be an upper bound of the increasing sequence, $\left\langle p_{\alpha} \mid \alpha<\theta\right\rangle$. By Claim 1.8.6, Definition 1.8.7, Claim 1.8.9 and clauses (a) through (c) of the induction, $p$ is an upper bound of the increasing sequence $\left\langle q_{g \mid Z_{n}} \mid n<\omega\right\rangle$, and so by Claim 1.8.10 and clause (a) of the induction, $p \Vdash \check{\tau} \in \dot{E} \cap \dot{S}$.

1.2. Applications. Utilizing the poset from the previous subsection and the existence of a supercompact cardinal, we now consider three models in which diamond fails on a set that reflects stationarily often.

Theorem 1.9. It is relatively consistent with the existence of a supercompact cardinal that all of the following hold simultaneously:

(1) $\mathrm{GCH}$;

(2) $\mathrm{AP}_{\aleph_{\omega}}$;

(3) $\operatorname{Refl}_{\aleph_{\omega}}$;

(4) $\diamond_{S}$ fails for some stationary $S \subseteq E_{\omega}^{\aleph_{\omega+1}}$.

Proof. We take as our ground model the model from [7, §5]. That is, GCH holds, $\kappa$ is a supercompact cardinal, there exists $\left\langle C_{\alpha} \mid \alpha<\kappa^{+\omega+1}\right\rangle$ which is a very weak square sequence, and there exists $\left\langle D_{\alpha} \mid \alpha \in E_{\Sigma_{\kappa}}^{\kappa^{+\omega+1}}\right\rangle$ which is a partial square sequence. We shall not define these concepts here; instead, we just mention two important facts. The first is that the properties of these sequences are indestructible under cofinality-preserving forcing which are $\omega$-distributive. The second is that in the generic extension by the Lévy collapse, $\operatorname{Col}\left(\omega_{1},<\kappa\right)$, these two sequences are combined to witness $\mathrm{AP}_{\aleph_{\omega}}$.

Let $\mathbb{P}$ denote the iteration of length $\kappa+1$ with backward Easton support, where for every inaccessible $\alpha \leq \kappa$, we force with $\mathbb{Q}\left(\alpha^{+\omega+1}\right)$ from Definition 1.4 and for accessible $\alpha<\kappa$, we use trivial forcing.

Let $G$ be $\mathbb{P}$-generic over $V$. Then by Lemmas 1.6, 1.7, and a well-known argument of Silver (see [1, §11]), $\kappa$ remains supercompact in $V[G]$. Also, the very weak square sequence and the partial square sequence remain as such.

By Theorem [1.8, there exists in $V[G]$ a stationary subset $S \subseteq E_{\omega}^{\kappa^{+\omega+1}}$ such that $\diamond_{S}$ fails. Finally, let $H$ be $\operatorname{Col}\left(\omega_{1},<\kappa\right)$-generic over $V[G]$. Work in $V[G][H]$. Then $\aleph_{2}=\kappa, \aleph_{\omega}=\kappa^{+\omega}, \aleph_{\omega+1}=\kappa^{+\omega+1}$, and $\mathrm{GCH}+\mathrm{AP}_{\aleph_{\omega}}$ holds. Since $\operatorname{Col}\left(\omega_{1},<\kappa\right)$ satisfies the $\kappa$-c.c., $S$ remains stationary, and $\nabla_{S}$ still fails (for if $\left\{\dot{A_{\delta}} \mid \delta \in S\right\}$ is a name for a $\nabla_{S}$-sequence in $V[G][H]$, then $\left\{\mathcal{A}_{\delta}:=\left\{A \subseteq \delta \mid \exists p \in \operatorname{Col}\left(\omega_{1},<\kappa\right)(p \Vdash\right.\right.$ $\left.\left.\left.\check{A}=\dot{A}_{\delta}\right)\right\} \mid \delta \in S\right\}$ would be a $\diamond_{S}^{-}$-sequence in $V[G]$; see [12]).

Finally, since $\kappa$ is $\kappa^{+\omega+1}$-supercompact in $V[G]$, an argument of Shelah yields that Refl $\aleph_{\omega}$ holds (see [1, §10]).

The next theorem shows that it is possible to have the failure of $\nabla_{S}$ for a set $S$ which reflects in an even stronger sense. 
Theorem 1.10. It is relatively consistent with the existence of a supercompact cardinal that there exists a singular cardinal $\lambda$ for which all of following hold simultaneously:

(1) $\lambda$ is a strong limit of countable cofinality and $2^{\lambda}=\lambda^{+}$;

(2) there exists a stationary $S \subseteq E_{\mathrm{cf}(\lambda)}^{\lambda^{+}}$such that:

(a) $\left\{\alpha \in E_{>\omega}^{\lambda^{+}} \mid S \cap \alpha\right.$ contains a club in $\left.\alpha\right\}$ is stationary;

(b) $\diamond_{S}$ fails.

Proof. Start with a model of MM. Put $\lambda:=\beth_{\omega}$. Since $\lambda$ is a singular strong limit, we get from [5] that $2^{\lambda}=\lambda^{+}$, and hence $\mathbb{Q}\left(\lambda^{+}\right)$is well defined, so let us work in the generic extension, $V^{\mathbb{Q}\left(\lambda^{+}\right)}$.

Since $\mathbb{Q}\left(\lambda^{+}\right)$is $\aleph_{2}$-directed closed, we get from Larson's theorem [13] that MM is preserved, and by the additional "good properties" of $\mathbb{Q}\left(\lambda^{+}\right)$, the cardinal structure is preserved as well. Then $\lambda=\beth_{\omega}, 2^{\lambda}=\lambda^{+}$, and there exists a stationary $S \subseteq E_{\mathrm{cf}(\lambda)}^{\lambda^{+}}$ such that $\nabla_{S}$ fails. Finally, clause (a) is an immediate consequence of the fact that MM implies Friedman's problem (see [5]).

Analysis of the models from Theorems 1.9 and 1.10 yields that these models satisfy a certain strong form of reflection, namely, $\operatorname{Refl}^{*}\left(\left[\lambda^{+}\right]^{\omega}\right)$, and hence there exists no very good scale (or even a better scale) for $\lambda$ in these models. We now consider a third model, establishing that a very good scale has no effect on the validity of diamond for reflecting stationary sets.

Theorem 1.11. It is relatively consistent with the existence of a supercompact cardinal that there exists a singular cardinal $\kappa$ for which all of following hold simultaneously:

(1) $\kappa$ is a strong limit of countable cofinality and $2^{\kappa}=\kappa^{+}$;

(2) there exists a very good scale for $\kappa$;

(3) $\nabla_{S}$ fails for some $S \subseteq E_{\mathrm{cf}(\kappa)}^{\kappa^{+}}$that reflects stationarily often.

Proof. Start with a model of $\mathrm{GCH}$ in which there exists a supercompact cardinal, $\kappa$. Let $\lambda:=\kappa^{+\omega}$.

Step 1. Let $\mathbb{P}_{1}$ denote the iteration of length $\kappa+1$ with backward Easton support, where for every inaccessible $\alpha \leq \kappa$, we force with $\mathbb{Q}\left(\alpha^{+\omega+1}\right)$, and for accessible $\alpha<\kappa$, we use trivial forcing. Let $V_{1}$ denote the generic extension by $\mathbb{P}_{1}$. Then, in $V_{1}, \mathrm{GCH}$ holds, $\kappa$ is supercompact, and there exists a stationary $S \subseteq E_{\mathrm{cf}(\lambda)}^{\lambda^{+}}$on which $\diamond_{S}$ fails.

Step 2. Work in $V_{1}$. Fix a normal ultrafilter $\mathcal{U}$ over $\mathcal{P}_{\kappa}\left(\lambda^{+}\right)$and the corresponding embedding $j: V_{1} \rightarrow M \simeq \operatorname{Ult}\left(V_{1}, \mathcal{U}\right)$. Let $\mathbb{P}_{2}$ denote the iteration of length $\kappa+1$ with backward Easton support, where for every inaccessible $\alpha \leq \kappa$, we force with $\operatorname{Add}\left(\alpha, \alpha^{+\omega+1}\right)$, adding $\alpha^{+\omega+1}$-Cohen functions from $\alpha$ to $\alpha$, and for accessible $\alpha<\kappa$, we use trivial forcing.

Let $G$ be $\mathbb{P}_{2}$-generic over $V_{1}$, and work in $V_{2}:=V_{1}[G]$. Then, by standard arguments (see [1, §11]), we may find a set $G^{*}$ such that the embedding $j$ lifts to an embedding $j^{*}: V_{1}[G] \rightarrow M\left[G^{*}\right]$. Let $\left\langle F_{\alpha}: \kappa \rightarrow \kappa \mid \alpha<\lambda^{+}\right\rangle$denote the generic functions introduced by the $\kappa_{t h}$-stage of $\mathbb{P}_{2}$. As in [8], we may choose $G^{*}$ in such a way that $F_{j^{*}(\alpha)}(\kappa)=\alpha$ for all $\alpha<\lambda^{+}$. Thus, $2^{\kappa}=2^{\lambda}=\lambda^{+}$. 
As $\mathbb{P}_{2}$ has the $\kappa^{+}$-c.c., $S$ remains stationary, and $\diamond_{S}$ still fails. (By the chain condition of $\mathbb{P}_{2}$, if $\nabla_{S}$ holds in $V_{2}$, then $\diamond_{S}^{-}$holds in $V_{1}$. However, $\diamond_{S}^{-}$entails $\diamond_{S}$, and the latter fails in $V_{1}$.) Let $T:=\left\{\alpha \in E_{<\kappa}^{\lambda^{+}} \mid \operatorname{cf}(\alpha)>\omega, S \cap \alpha\right.$ is stationary $\}$. Since $S$ is a stationary subset of $E_{<\kappa}^{\lambda^{+}}$and $\kappa$ is $\lambda^{+}$-supercompact (as witnessed by $\left.j^{*}\right)$, the set $T$ is stationary.

Note that the cardinal structure in $V_{2}$ is the same as in $V$.

Step 3. Work in $V_{2}$. Let $\mathcal{U}^{*}:=\left\{X \subseteq \mathcal{P}_{\kappa}\left(\kappa^{+\omega+1}\right) \mid j^{*}\right.$ " $\left.\lambda^{+} \in j^{*}(X)\right\}$. Then $\mathcal{U}^{*}$ is a normal ultrafilter extending $\mathcal{U}$. For every $n<\omega$, let $\mathcal{U}_{n}$ denote the projection of $\mathcal{U}^{*}$ to $\mathcal{P}_{\kappa}\left(\kappa^{+n}\right)$. Next, let $\left\langle\mathcal{Q}, \leq, \leq^{*}\right\rangle$ denote the variation of supercompact Prikry forcing from [8, Definition 2.9]. That is, instead of working with a single measure, we work with the sequence of measures $\left\langle\mathcal{U}_{n} \mid n<\omega\right\rangle$. By [요, §2], we have:

(a) $\langle\mathcal{Q}, \leq\rangle$ satisfies the $\lambda^{+}$-c.c.;

(b) $\langle\mathcal{Q}, \leq\rangle$ does not add new bounded subsets to $\kappa$;

(c) in the generic extension by $\langle\mathcal{Q}, \leq\rangle,\left(\kappa^{+n}\right)^{V}$ changes its cofinality to $\omega$, for every $n<\omega$.

Let $V_{3}$ denote the generic extension by $\langle\mathcal{Q}, \leq\rangle$. Work in $V_{3}$. Then $\kappa$ is a strong limit cardinal of countable cofinality and $2^{\kappa}=\left(\lambda^{+}\right)^{V_{2}}=\kappa^{+}$. Since $\langle\mathcal{Q}, \leq\rangle$ has the $\lambda^{+}$-c.c., the sets $S$ and $T$ remain stationary subset of $\kappa^{+}$, and $\nabla_{S}$ still fails.

Claim 1.11.1. $S$ reflects stationarily often.

Proof. Recall that we work in $V_{3}$. Put $T^{*}:=\left\{\alpha<\kappa^{+} \mid \operatorname{cf}(\alpha)>\omega, S \cap \alpha\right.$ is stationary $\}$. Since $T$ is stationary, to show that $S$ reflects stationarily often, it suffices to establish that $T \subseteq T^{*}$. For this, it suffices to prove that if $\alpha \in E_{>\omega}^{\kappa^{+}} \cap E_{<\kappa}^{\kappa^{+}}$and $C$ is a club subset of $\alpha$ then there exists a club $C^{\prime} \subseteq C$ lying in $V_{2}$. But this is obvious: fix, in $V_{2}$, a continuous function $\pi: \mathrm{cf}^{V_{2}}(\alpha) \rightarrow \alpha$ whose image is cofinal in $\alpha$. Put $C^{\prime}:=C \cap \operatorname{Im}(\pi)$. Then $C^{\prime}$ is a club subset of $C$ and $\pi^{-1}\left[C^{\prime}\right]$ is a club subset of $\mathrm{cf}^{V_{2}}(\alpha)$. By property (c) and $\alpha \in E_{>\omega}^{\kappa^{+}} \cap E_{<\kappa}^{\kappa^{+}}$, we have $\mathrm{cf}^{V_{2}}(\alpha)=\mathrm{cf}^{V_{3}}(\alpha)$, so property (b) entails that $\pi^{-1}\left[C^{\prime}\right]$ is in $V_{2}$, and hence $C^{\prime}$ is in $V_{2}$, as requested.

Claim 1.11.2. There exists a very good scale for $\kappa$.

Proof. Let $\left\langle P_{n} \mid n<\omega\right\rangle$ denote the supercompact Prikry sequence introduced by $\langle\mathcal{Q}, \leq\rangle$ over $V_{2}$. For each $n<\omega$, consider the inaccessible cardinal $\kappa_{n}=P_{n} \cap \kappa$. For each $\alpha<\kappa^{+}=\left(\lambda^{+}\right)^{V_{2}}$, define a function $t_{\alpha} \in \prod_{n<\omega} \kappa_{n}^{+\omega+1}$ as follows:

$$
t_{\alpha}(n):=\left\{\begin{array}{ll}
F_{\alpha}\left(\kappa_{n}\right), & F_{\alpha}\left(\kappa_{n}\right)<\kappa_{n}^{+\omega+1} \\
0, & \text { otherwise }
\end{array} \quad(n<\omega) .\right.
$$

Then, by Proposition 2.21 of $[8],\left\langle t_{\alpha} \mid \alpha<\kappa^{+}\right\rangle$is a very good scale.

1.3. Uncountable cofinality. It is worth mentioning that via a straightforward modification to the proofs of subsection 1.1 it is possible to handle singular cardinals of uncountable cofinality as well. More specifically, we have:

Theorem 1.12. Suppose $\lambda$ is a strong limit singular cardinal, and $2^{\lambda}=\lambda^{+}$Then there exists a notion of forcing $\mathbb{Q}^{\prime}\left(\lambda^{+}\right)$, satisfying:

(1) $\mathbb{Q}^{\prime}\left(\lambda^{+}\right)$is $\lambda^{+}$-directed closed;

(2) $\mathbb{Q}^{\prime}\left(\lambda^{+}\right)$has the $\lambda^{++}$-c.c.; 
(3) $\left|\mathbb{Q}^{\prime}\left(\lambda^{+}\right)\right|=\lambda^{++}$;

(4) in $V^{\mathbb{Q}^{\prime}\left(\lambda^{+}\right)}, \diamond_{S}$ fails for some stationary $S \subseteq E_{\mathrm{cf}(\lambda)}^{\lambda^{+}}$.

Remark. In particular, it is possible to obtain the failure of diamond on a stationary subset of $\lambda^{+}$in the presence of a supercompact cardinal in the interval $(\operatorname{cf}(\lambda), \lambda)$.

Outline of the proof of Theorem 1.12 . $\mathbb{Q}^{\prime}\left(\lambda^{+}\right)$is a dense subset of the forcing notion for injecting a Cohen subset of $E_{\mathrm{cf}(\lambda)}^{\lambda^{+}}$followed by the iteration for killing all potential diamond sequences over it. To argue that the generic Cohen set remains stationary, one takes an elementary submodel $M \prec\left\langle H(\chi),<_{\chi}\right)$ with $\tau=M \cap \lambda^{+} \in E_{\operatorname{cf}(\lambda)}^{\lambda^{+}}$and $<\operatorname{cf}(\lambda) M \subseteq M$, that contains all the relevant information including a name for a given club. We then collect enough incomparable conditions that eventually decide the identity of $\operatorname{cf}(\lambda)$ many elements of the club below $\tau$, aiming for Claims 1.8.10 and 1.8.11

As in the original construction, this is achieved by recursively defining a continuous sequence of the form $\left\langle\left(\gamma_{\alpha}, Y_{\alpha}, Z_{\alpha}, \mathcal{F}_{\alpha}\right) \mid \alpha<\operatorname{cf}(\lambda)\right\rangle$, where the successor and base steps involve the construction of an upper triangular matrix of conditions.

Remark. It follows from the proof in [15, $\S 4]$ that the above $\mathbb{Q}^{\prime}\left(\lambda^{+}\right)$is not isomorphic to Cohen's notion of forcing, $\operatorname{Add}\left(\lambda^{+}, \lambda^{++}\right)$.

\section{Negation of Guessing}

In [4, Džamonja and Shelah considered a particular consequence of diamond and established the consistency of its negation. To state their result, we need the following two definitions.

Definition 2.1. For a function $f: \lambda^{+} \rightarrow \operatorname{cf}(\lambda)$, let $\kappa_{f}$ denote the minimal cardinality of a family $\mathcal{P} \subseteq\left[\lambda^{+}\right]^{\operatorname{cf}(\lambda)}$ with the property that whenever $Z \subseteq \lambda^{+}$is such that $\bigwedge_{\beta<\operatorname{cf}(\lambda)}\left|Z \cap f^{-1}\{\beta\}\right|=\lambda^{+}$, then there exist some $a \in \mathcal{P}$ with $\sup (f[a \cap Z])=\operatorname{cf}(\lambda)$.

It is obvious that the function $f: \lambda^{+} \rightarrow\{0\}$ satisfies $\kappa_{f}=0$. Also notice that any partition of $\lambda^{+}$into $\operatorname{cf}(\lambda)$ many sets of cardinality $\lambda^{+}$induces a non-trivial function, that is, a function $f \in{\lambda^{+}}^{+} \operatorname{cf}(\lambda)$ with $\kappa_{f}>0$.

Definition 2.2. For a singular cardinal $\lambda$, we say that $\lambda^{+}$-guessing holds iff $\kappa_{f} \leq$ $\lambda^{+}$for all $f \in{\lambda^{+}}^{+} \operatorname{cf}(\lambda)$.

We refer the reader to [4] for background and motivation for this definition, but let us just mention the obvious fact that $\diamond_{\lambda^{+}}$implies $\lambda^{+}$-guessing. In [4, §2], the consistency of the negation of $\lambda^{+}$-guessing has been established:

Theorem 2.3 (Džamonja-Shelah). It is relatively consistent with the existence of a supercompact cardinal that there exist a strong limit singular cardinal $\lambda$ and a function $f: \lambda^{+} \rightarrow \operatorname{cf}(\lambda)$ such that $\kappa_{f}=2^{\lambda}>\lambda^{+}$.

Here, we reduce the large cardinal hypothesis significantly by establishing that any model with a strong limit singular cardinal $\lambda$ with $2^{\lambda}>\lambda^{+}$will do. Moreover, in such a model, any non-trivial function is a counterexample.

Theorem 2.4. If $\lambda$ is a strong limit singular cardinal, then

$$
\left\{\kappa_{f} \mid f \in \lambda^{\lambda^{+}} \operatorname{cf}(\lambda)\right\}=\left\{0,2^{\lambda}\right\} .
$$


Proof. Since $\lambda$ is a strong limit, the next lemma tells us that any non-trivial function, $f$, satisfies $\kappa_{f}=\lambda^{\mathrm{cf}(\lambda)}=2^{\lambda}$.

Lemma 2.5. Suppose $\lambda$ is an infinite cardinal, and $2^{\operatorname{cf}(\lambda)}+\lambda^{<\operatorname{cf}(\lambda)} \leq \lambda^{+}$. Then $\kappa_{f}=\lambda^{\mathrm{cf}(\lambda)}$ for every non-trivial function $f \in \lambda^{\lambda^{+}} \operatorname{cf}(\lambda)$.

Proof. Suppose $f: \lambda^{+} \rightarrow \operatorname{cf}(\lambda)$ is a function with $\kappa_{f}>0$. Fix $\beta<\operatorname{cf}(\lambda)$, and let $A_{\beta}:=\left\{\delta<\lambda^{+} \mid f(\delta)=\beta\right\}$. Since $\kappa_{f}>0$, we have $\left|A_{\beta}\right|=\lambda^{+} \geq \lambda^{<\operatorname{cf}(\lambda)}$, so let us fix a surjection $\psi_{\beta}: A_{\beta} \rightarrow^{<\operatorname{cf}(\lambda)} \lambda$ such that $\left(\psi_{\beta}\right)^{-1}\{\eta\}$ has cardinality $\lambda^{+}$for all $\eta \in\left\langle\operatorname{cf}(\lambda) \lambda\right.$. Clearly, if $\mathcal{P} \subseteq\left[\lambda^{+}\right]^{\operatorname{cf}(\lambda)}$ is a family of size $<\lambda^{+}$, then there exists a set $Z \subseteq \lambda^{+}$such that $\left|Z \cap A_{\beta}\right|=\lambda^{+}$for all $\beta<\lambda^{+}$, while $Z \cap a=\emptyset$ for all $a \in \mathcal{P}$. This shows that $\kappa_{f} \geq \lambda^{+}$.

Next, for every function $g \in{ }^{\operatorname{cf}(\lambda)} \lambda$, put $Z_{g}:=\left\{\delta<\lambda^{+} \mid g\left\lceil f(\delta)=\psi_{f(\delta)}(\delta)\right\}\right.$.

Claim 2.5.1. $\bigwedge_{\beta<\operatorname{cf}(\lambda)}\left|Z_{g} \cap f^{-1}\{\beta\}\right|=\lambda^{+}$for every $g \in{ }^{\operatorname{cf}(\lambda)} \lambda$.

Proof. For every $\beta<\operatorname{cf}(\lambda)$, letting $\eta:=g\left\lceil\beta\right.$, we get that $Z_{g} \cap f^{-1}\{\beta\}=\{\delta \in$ $A_{\beta} \mid g\left\lceil f(\delta)=\psi_{f(\delta)}(\delta)\right\}=\left\{\delta \in A_{\beta} \mid \eta=\psi_{\beta}(\delta)\right\}=\left(\psi_{\beta}\right)^{-1}\{\eta\}$.

Let $\left\{g_{i} \mid i<\lambda^{\operatorname{cf}(\lambda)}\right\}$ be an injective enumeration of ${ }^{\operatorname{cf}(\lambda)} \lambda$. By $\lambda^{+} \leq \kappa_{f} \leq \lambda^{\operatorname{cf}(\lambda)}$, we avoid trivialities and assume that $\lambda^{\mathrm{cf}(\lambda)}>\lambda^{+}$. Thus, it suffices to establish the following.

Claim 2.5.2. For all $a \in\left[\lambda^{+}\right]^{\operatorname{cf}(\lambda)}, I_{a}:=\left\{i<\lambda^{\operatorname{cf}(\lambda)} \mid \sup \left(f\left[a \cap Z_{g_{i}}\right]\right)=\operatorname{cf}(\lambda)\right\}$ has cardinality $\leq \lambda^{+}$.

Proof. Assume towards a contradiction that $a \in[\lambda]^{\mathrm{cf}(\lambda)}$ is such that $\left|I_{a}\right|>\lambda^{+}$. By $\left|I_{a}\right| \geq\left(2^{\operatorname{cf}(\lambda)}\right)^{+}$and the Erdös-Rado theorem, let us pick a set $I^{\prime} \subseteq I_{a}$ with $\left|I^{\prime}\right|>\operatorname{cf}(\lambda)$ and an ordinal $\gamma<\operatorname{cf}(\lambda)$ such that $g_{i_{0}}(\gamma) \neq g_{i_{1}}(\gamma)$ for all distinct $i_{0}, i_{1} \in I^{\prime}$. Shrinking further, pick $I^{\prime \prime} \subseteq I^{\prime}$ with $\left|I^{\prime \prime}\right|>\operatorname{cf}(\lambda)$ and an ordinal $\beta>\gamma$ such that $\beta=\min \left(f\left[a \cap Z_{g_{i}}\right]\right)$ for all $i \in I^{\prime \prime}$. Finally, for all $i \in I^{\prime \prime}$, pick $\delta_{i} \in a \cap Z_{g_{i}}$ such that $f\left(\delta_{i}\right)=\beta$. Since $|a|=\operatorname{cf}(\lambda)<\left|I^{\prime \prime}\right|$, we may find distinct $i_{0}, i_{1} \in I^{\prime \prime}$ for which $\delta_{i_{0}}=\delta_{i_{1}}$.

Finally, by $\delta_{i_{0}} \in Z_{g_{i_{0}}}, \delta_{i_{1}} \in Z_{g_{i_{1}}}$ and $\delta_{i_{0}}=\delta_{i_{1}}$, we have

$$
g_{i_{0}} \uparrow \beta=g_{i_{0}} \uparrow f\left(\delta_{i_{0}}\right)=\psi_{\delta_{i_{0}}}\left(\delta_{i_{0}}\right)=\psi_{\delta_{i_{1}}}\left(\delta_{i_{1}}\right)=g_{i_{1}} \uparrow f\left(\delta_{i_{1}}\right)=g_{i_{1}} \uparrow \beta,
$$

which contradicts the existence of some $\gamma<\beta$ with $g_{i_{0}}(\gamma) \neq g_{i_{1}}(\gamma)$.

By combining the arguments of the above proof with the ones from [14, it is possible to obtain a lower bound on $\kappa_{f}$ even without assuming $2^{\operatorname{cf}(\lambda)} \leq \lambda^{+}$. Namely, if $\lambda$ is a singular cardinal and $\lambda^{<\operatorname{cf}(\lambda)} \leq \lambda^{+}$, then $\kappa_{f} \geq \operatorname{pp}(\lambda)$ for every non-trivial function $f \in{ }^{\lambda^{+}} \operatorname{cf}(\lambda)$. In particular, if $\lambda>\operatorname{cf}(\lambda)=\omega$, then $\kappa_{f} \geq \operatorname{pp}(\lambda)$ for every non-trivial $f$. The corollary follows:

Corollary 2.6. The following are equivalent:

(1) Shelah's strong hypothesis;

(2) $\lambda^{+}$-guessing holds for all singular cardinal $\lambda$. 


\section{The sup-FunCtion on Stationary subsets of $\left[\lambda^{+}\right]^{\omega}$}

In this section, we shall supply a negative answer to the following question.

Question (König-Larson-Yoshinobu, 11). Let $\lambda>\omega_{1}$ be a successor cardinal. Is it possible to prove in ZFC that every stationary $\mathcal{B} \subseteq[\lambda]^{\aleph_{0}}$ can be thinned out to a stationary $\mathcal{A} \subseteq \mathcal{B}$ on which the sup-function is $1-1$ ?

We refer the reader to [11 for motivation and background concerning this question. Recall that a set $\mathcal{A} \subseteq \mathcal{P}(\lambda)$ is said to be stationary (in the generalized sense) iff for every function $f:[\lambda]^{<\omega} \rightarrow \lambda$, there exists some $A \in \mathcal{A}$ such that $f$ " $[A]^{<\omega} \subseteq A$. Now, it is obvious that if $\operatorname{cf}\left([\lambda]^{\omega}, \subseteq\right)>\lambda$, then $\mathcal{B}:=[\lambda]^{\omega}$ is a counterexample to the above question. In particular, any model on which the singular cardinals hypothesis fails gives a negative answer. Thus, in this section, we shall focus on answering the above question in the context of $\mathrm{GCH}$.

Definition 3.1. Given a set $\mathcal{X} \subseteq \mathcal{P}(\lambda)$, denote $S(\mathcal{X}):=\{\sup (X) \mid X \in \mathcal{X}\}$.

Definition 3.2. For an infinite cardinal $\lambda$ and a stationary set $S \subseteq E_{<\lambda}^{\lambda^{+}}$, consider the following three principles:

(a) $(1)_{S}$ asserts that there exists a stationary $\mathcal{X} \subseteq\left[\lambda^{+}\right]^{<\lambda}$ such that:

- the sup-function on $\mathcal{X}$ is 1-to-1;

- $S(\mathcal{X}) \subseteq S$.

(b) $(\lambda)_{S}$ asserts that there exists a stationary $\mathcal{X} \subseteq\left[\lambda^{+}\right]^{<\lambda}$ such that:

- the sup-function on $\mathcal{X}$ is $(\leq \lambda)$-to- 1 ;

- $S(\mathcal{X}) \subseteq S$.

(c) $\boldsymbol{\alpha}_{S}^{-}$asserts that there exists a sequence $\left\langle\mathcal{A}_{\delta} \mid \delta \in S\right\rangle$ such that:

- for all $\delta \in S, \mathcal{A}_{\delta} \subseteq[\delta]^{<\lambda}$ and $\left|\mathcal{A}_{\delta}\right| \leq \lambda$;

- if $Z$ is a cofinal subset of $\lambda^{+}$, then the following set is stationary:

$$
\left\{\delta \in S \mid \exists A \in \mathcal{A}_{\delta}(\sup (A \cap Z)=\delta)\right\} .
$$

The principle $\boldsymbol{\alpha}_{S}^{-}$has been considered in [15] and was found to be the $\mathrm{GCH}$-free version of $\diamond_{S}$. From this, we easily get the following.

Lemma 3.3. Suppose $\lambda$ is an infinite cardinal and $S \subseteq E_{<\lambda}^{\lambda^{+}}$is stationary.

Then $(1) \Rightarrow(2) \Rightarrow(3) \Rightarrow(4)$, and if $2^{\lambda}=\lambda^{+}$, then moreover, (4) $\Rightarrow(1)$, where:

(1) $\diamond_{S}$;

(2) $(1)_{S}$;

(3) $(\lambda)_{S}$;

(4) $\boldsymbol{\phi}_{S}^{-}$.

Proof. (1) $\Rightarrow(2)$ By $\diamond_{S}$, pick a collection $\left\{f_{\delta}:[\delta]^{<\omega} \rightarrow \delta \mid \delta \in S\right\}$ such that for every $f:\left[\lambda^{+}\right]^{<\omega} \rightarrow \lambda^{+}$there exists some $\delta \in S$ with $f\left\lceil[\delta]^{<\omega}=f_{\delta}\right.$. For each $\delta \in S$, pick a cofinal $Y_{\delta} \subseteq \delta$ of minimal order-type and find $X_{\delta} \supseteq Y_{\delta}$ with $\left|X_{\delta}\right|=\left|Y_{\delta}\right|$ such that $f_{\delta}$ " $\left[X_{\delta}\right]^{<\omega} \subseteq X_{\delta}$. It is now easy to see that $\mathcal{X}:=\left\{X_{\delta} \mid \delta \in S\right\}$ is as requested.

$(2) \Rightarrow(3)$ is obvious.

$(3) \Rightarrow(4)$ Let $\mathcal{X}$ exemplify $(\lambda)_{S}$. For each $\delta \in S$, let $\mathcal{A}_{\delta}:=\{X \in \mathcal{X} \mid \sup (X)=$ $\delta\}$. To see that $\left\langle\mathcal{A}_{\delta} \mid \delta \in S\right\rangle$ witness $\boldsymbol{\alpha}_{S}^{-}$, we fix a cofinal subset $Z \subseteq \lambda^{+}$and a club $C \subseteq \lambda^{+}$, and argue that there exists $\delta \in C \cap S$ and $A \in \mathcal{A}_{\delta}$ such that $\sup (Z \cap A)=\delta$. Define a function $f:\left[\lambda^{+}\right]^{<\omega} \rightarrow \lambda^{+}$as follows:

$$
f(\sigma):= \begin{cases}\min (Z \backslash \sup (\sigma)), & |\sigma| \text { is odd, } \\ \min (C \backslash \sup (\sigma)), & |\sigma| \text { is even. }\end{cases}
$$


Since $\mathcal{X}$ is stationary, we may pick some $X \in \mathcal{X}$ such that $f$ " $[X]^{<\omega} \subseteq X$. Put $\delta:=\sup (X)$. Then $X \in \mathcal{A}_{\delta}, f^{\prime \prime}[X]^{7}$ is a cofinal subset of $Z \cap \delta$, and $f$ " $[X]^{4}$ is a cofinal subset of $C \cap \delta$. In particular, $\sup (X \cap Z)=\sup (X \cap C)=\delta$, so $\delta \in C$ and we are done.

Finally, if $2^{\lambda}=\lambda^{+}$, then a theorem from [15] stating that $\diamond_{S} \Leftrightarrow 2^{\lambda}=\lambda^{+}+\boldsymbol{\alpha}_{S}^{-}$ yields $(4) \Rightarrow(1)$.

Remark. The preceding lemma improves an unpublished result by Matsubara and Sakai, who established the implication $(3) \Rightarrow(1)$ under stronger cardinal arithmetic assumptions.

Since, under mild cardinal arithmetic hypothesis, the above principles coincide, it is interesting to study whether these principles can be separated. The reader who is only interested in the promised solution to the above-mentioned question may now skip to Theorem 3.6 below.

Proposition 3.4. If the singular cardinals hypothesis fails, then for some singular cardinal $\lambda$, $\boldsymbol{\beta}_{S}^{-}$holds, while $(\lambda)_{S}$ fails, for $S:=E_{\neq \mathrm{cf}(\lambda)}^{\lambda^{+}}$

Proof. By [15], $\boldsymbol{\alpha}_{E_{\neq \mathrm{cf}(\lambda)}^{-}+}^{+}$holds for every infinite cardinal $\lambda$, so let us focus on the second component. Suppose that the singular cardinals hypothesis fails. By a theorem of Shelah [18, in this case, there exists a singular cardinal $\lambda$ such that $\operatorname{cf}(\lambda)=\omega$ and $\operatorname{cov}\left(\lambda, \lambda, \operatorname{cf}(\lambda)^{+}, 2\right)>\lambda^{+}$. This means that for every $\mathcal{X} \subseteq[\lambda]^{<\lambda}$ of size $\lambda^{+}$, there exists some $x \in[\lambda]^{\omega}$ such that $x \nsubseteq X$ for all $X \in \mathcal{X}$. Now assume towards a contradiction that $\mathcal{X}$ witnesses $(\lambda)_{E_{\neq \mathrm{cf}(\lambda)}^{\lambda+}}$. In particular, $\mathcal{X} \subseteq\left[\lambda^{+}\right]^{<\lambda}$ and $|\mathcal{X}|=\lambda^{+}$. Pick a function $x: \omega \rightarrow \lambda$ with $\operatorname{Im}(x) \nsubseteq X$ for all $X \in \mathcal{X}$. Define $f:\left[\lambda^{+}\right]^{<\omega} \rightarrow \lambda^{+}$by $f(\sigma):=x(|\sigma|)$ for all $\sigma \in\left[\lambda^{+}\right]^{<\omega}$. Since $\mathcal{X}$ is stationary, there exists some $X \in \mathcal{X}$ such that $f "[X]^{<\omega} \subseteq X$. In particular, $\operatorname{Im}(x) \subseteq X$, contradicting the choice of $x$.

Proposition 3.5. $(1)_{S} \nRightarrow \diamond_{S}$ for any uncountable cardinal $\lambda$ and any stationary $S \subseteq E_{\omega}^{\lambda^{+}}$.

Proof. Suppose $\lambda$ is an uncountable cardinal, $S \subseteq E_{\omega}^{\lambda^{+}}$and $(1)_{S}$ holds, as witnessed by a stationary set $\mathcal{X}$. Use Cohen forcing to blow up the continuum above $\lambda^{+}$; then $\diamond_{S}$ fails. Finally, since Cohen forcing is proper, $\mathcal{X}$ remains stationary, so it still witnesses $(1)_{S}$.

Answering the above-mentioned question in the negative, we now prove:

Theorem 3.6. It is relatively consistent with $\mathrm{ZFC}$ that the $\mathrm{GCH}$ holds and there exists a stationary subset $\mathcal{B} \subseteq\left[\aleph_{\omega+1}\right]^{\omega}$ that cannot be thinned out to a stationary $\mathcal{A} \subseteq \mathcal{B}$ on which the sup-function is injective.

Proof. Start with a model of GCH in which $\diamond_{S}$ fails for some stationary $S \subseteq E_{\omega}^{\aleph_{\omega}}$ (by appealing to the forcing from $[17, \S 2]$ or by forcing with $\mathbb{Q}\left(\aleph_{\omega+1}\right)$ from section 11).

Claim 3.6.1. $\mathcal{B}:=\left\{X \in\left[\aleph_{\omega+1}\right]^{\omega} \mid \sup (X) \in S\right\}$ is stationary.

Proof. Suppose $f:\left[\aleph_{\omega+1}\right]^{<\omega} \rightarrow \aleph_{\omega+1}$ is a given function. Since $\left\{\delta \in \aleph_{\omega+1} \mid\right.$ $\left.f^{\prime \prime}[\delta]^{<\omega} \subseteq \delta\right\}$ is a club and $S$ is stationary, we may fix some $\delta \in S$ such that 
$f^{\prime \prime}[\delta]^{<\omega} \subseteq \delta$. Pick $Y \in[\delta]^{\omega}$ with $\sup (Y)=\delta$. Then there exists $X \supseteq Y$ such that $|X|=|Y|$ and $f^{\prime \prime}[X]^{<\omega} \subseteq X$. Such an $X$ is in $\mathcal{B}$, so we are done.

Now, suppose $\mathcal{A} \subseteq \mathcal{B}$ is stationary on which the sup-function is injective. Then $(1)_{S(\mathcal{A})}$ holds, and as $S(\mathcal{A}) \subseteq S$, also $(1)_{S}$ holds, contradicting Lemma 3.3 and the fact that $\nabla_{S}$ fails.

Let us emphasize that the above theorem does not require large cardinals. Assuming large cardinals, one can obtain a stronger counterexample:

Theorem 3.7. It is relatively consistent with the existence of a supercompact cardinal that the $\mathrm{GCH}$ holds and there exists a stationary subset $\mathcal{B} \subseteq\left[\aleph_{\omega+1}\right]^{\omega}$ which is large in the following two senses:

(1) if $X \in\left[\aleph_{\omega+1}\right]^{\omega}$ and $\sup (X) \in S(\mathcal{B})$, then $X \in \mathcal{B}$;

(2) for every stationary $\mathcal{A} \subseteq \mathcal{B}$, there exists some $X \in\left[\aleph_{\omega+1}\right]^{\omega_{1}}$ with $\omega_{1} \subseteq X$ and $\operatorname{cf}(\operatorname{otp}(X))=\omega_{1}$ such that $\mathcal{A} \cap[X]^{\omega}$ is stationary.

Still, the sup-function is not injective on any stationary subset of $\mathcal{B}$.

Proof. Consider the model from Theorem 1.9. GCH holds, and there exists a stationary subset $S \subseteq E_{\omega}^{\aleph_{\omega+1}}$ such that $\diamond_{S}$ fails. Put $\mathcal{B}:=\left\{X \in\left[\aleph_{\omega+1}\right]^{\omega} \mid \sup (X) \in\right.$ $S\}$. Then (1) is obvious, and by Lemma 3.3. the sup-function is not injective on any stationary subset of $\mathcal{B}$.

Finally, clause (2) follows from the general fact from [5] that if $\kappa$ is a supercompact cardinal, then $V^{\operatorname{Col}\left(\omega_{1},<\kappa\right)} \models \operatorname{Refl}^{*}\left(\left[\kappa^{+\omega+1}\right]^{\omega}\right)$.

Note that $\mathrm{SAP}_{\aleph_{\omega}}$ (and hence $\square_{\aleph_{\omega}}^{*}$ ) necessarily fails in a model consisting of such $\mathcal{B}$, hence, the high consistency strength.

Discussion. To obtain a finer understanding of Theorem 3.6, we now discuss a more direct argument which allows us to point our finger at the role of the injectivity of the sup-function.

We start with a model of $\mathrm{GCH}$ and let $\lambda:=\aleph_{\omega}$. We consider the forcing notion $\mathbb{X}\left(\lambda^{+}\right)$as an alternative to $\mathbb{S}\left(\lambda^{+}\right)$from Section 1, A condition $\mathcal{X}$ is in $\mathbb{X}\left(\lambda^{+}\right)$iff $\mathcal{X}$ : $[\alpha]^{\omega} \rightarrow 2$ for some $\alpha<\lambda^{+}$. A condition $\mathcal{X}^{\prime}$ is stronger than $\mathcal{X}$ iff $\mathcal{X}^{\prime} \supseteq \mathcal{X}$. To study the injectivity of the sup-function, we also consider $\mathbb{X}^{1}\left(\lambda^{+}\right)$, where $\mathcal{X} \in \mathbb{X}^{1}\left(\lambda^{+}\right)$iff $\mathcal{X} \in \mathbb{X}\left(\lambda^{+}\right)$and for all $\tau<\lambda^{+}$, the set $\left\{X \in\left[\lambda^{+}\right]^{\omega} \mid \mathcal{X}(X)=1 \& \sup (X)=\tau\right\}$ contains at most a single element.

Let $\dot{S}\left(\lambda^{+}\right):=\left\{\langle\sup (X), \mathcal{X}\rangle \mid X \in \mathcal{X} \in \mathbb{X}\left(\lambda^{+}\right) \& \mathcal{X}(X)=1\right\}$ be the canonical name for the generic subset of $E_{\omega}^{\lambda^{+}}$introduced by $\mathbb{X}\left(\lambda^{+}\right)$and by $\mathbb{X}^{1}\left(\lambda^{+}\right)$. Now, instead of forcing with $\mathbb{Q}\left(\lambda^{+}\right)=\mathbb{S}\left(\lambda^{+}\right) * \mathbb{K} \mathbb{A D}\left(\dot{S}\left(\lambda^{+}\right)\right)$, we shall force with $\mathbb{P}\left(\lambda^{+}\right):=$ $\mathbb{X}\left(\lambda^{+}\right) * \mathbb{K} \mathbb{A D}\left(\dot{S}\left(\lambda^{+}\right)\right)$. For the sake of our comparison, we also define $\mathbb{P}^{1}\left(\lambda^{+}\right):=$ $\mathbb{X}^{1}\left(\lambda^{+}\right) * \mathbb{K} \mathbb{A D}\left(\dot{S}\left(\lambda^{+}\right)\right)$

The same arguments as in Section 1 shows that $\mathbb{P}\left(\lambda^{+}\right)$and $\mathbb{P}^{1}\left(\lambda^{+}\right)$satisfy the $\lambda^{++}$-c.c. and that they have a dense subset in which every increasing sequence of conditions of length $<\lambda^{+}$has an upper bound.

We now sketch the changes to be made to the proof of Theorem 1.8 to show that if $G * H$ is $\mathbb{P}\left(\lambda^{+}\right)$-generic and $\mathcal{S}:=\left\{X \in\left[\lambda^{+}\right]^{\omega} \mid \exists \mathcal{X} \in G(X \in \operatorname{dom}(\mathcal{X}) \& \mathcal{X}(X)=1)\right\}$, then $V[G][H] \models \mathcal{S}$ is stationary. 
Instead of fixing a name for a club $E \subseteq \lambda^{+}$, we fix a name for a function $e:\left[\lambda^{+}\right]^{<\omega} \rightarrow \lambda^{+}$. Instead of deciding the value for $\min (E \backslash \alpha)$, we decide $e \uparrow$ $[\alpha]^{<\omega}$, utilizing the fact that $\mathbb{P}\left(\lambda^{+}\right)$does not add bounded subsets of $\lambda^{+}$. Then the analogue of Claim 1.8.10 is that for all $g \in{ }^{Z} 2$, if $q$ is an upper bound of the increasing sequence $\left\langle q_{g \mid Z_{n}} \mid n<\omega\right\rangle$, then $q$ forces that $\tau$ is a closure point of $e$ and, moreover, $q$ decides $e\left\lceil[\tau]^{<\omega}\right.$ to be, say, $e^{g}:[\tau]^{<\omega} \rightarrow \tau$.

While in Section 1, we didn't care about $E \cap \tau$ and only focused on the fact that $\tau$ is a (closure) point of $E$. Here we really need to know $e \uparrow[\tau]^{<\omega}$. Notice, however, that if $g_{0} \neq g_{1}$, then it is possible that $e^{g_{0}} \neq e^{g_{1}}$. This is a subtle point, and we shall get back to it at the end of the discussion.

From here, we continue smoothly until we reach to the construction of the sequence of conditions $\left\langle p_{\alpha}=\left\langle\mathcal{X}_{\alpha}, k_{\alpha}\right\rangle \mid \alpha<\theta\right\rangle$ and the chain of functions $\left\{h_{\alpha}\right.$ : $\left.Z \cap\left(\varepsilon_{\alpha}+1\right) \rightarrow 2 \mid \alpha<\theta\right\}$. At the induction base, instead of choosing $\left\langle s_{0}, \emptyset\right\rangle \in D_{0}$ with $\bar{s} \cup\{(\tau, 1)\} \sqsubseteq s_{0}$, we first pick an arbitrary cofinal subset $X \in[\tau]^{\omega}$ and then choose $\left\langle\mathcal{X}_{0}, \emptyset\right\rangle \in D_{0}$ such that $\mathcal{X}_{0} \supseteq \overline{\mathcal{X}} \cup\{(X, 1)\}$. Once the construction is completed, we let $g:=\bigcup_{\alpha<\theta} h_{\alpha}$ and let $\left\langle\mathcal{X}^{\prime}, k^{\prime}\right\rangle$ be an upper bound for the increasing sequence, $\left\langle p_{\alpha} \mid \alpha<\theta\right\rangle$.

Then $\left\langle\mathcal{X}^{\prime}, k^{\prime}\right\rangle$ is an upper bound of $\left\langle q_{g \mid Z_{n}} \mid n<\omega\right\rangle$, and hence decides $e \uparrow[\tau]^{<\omega}$ to be $e^{g}:[\tau]^{<\omega} \rightarrow \tau$. Pick a cofinal $X^{\prime} \subseteq[\tau]^{\omega}$ which is closed under $e^{g}$. Then $\left\langle\mathcal{X}^{\prime} \cup\left\{\left(X^{\prime}, 1\right)\right\} \backslash\left\{\left(X^{\prime}, 0\right)\right\}, k^{\prime}\right\rangle$ is a legitimate condition because $\sup \left(X^{\prime}\right)=\tau$ is already forced by $\left\langle\mathcal{X}_{0}, k_{0}\right\rangle$ to be an element of $S\left(\lambda^{+}\right)$, and it moreover forces that

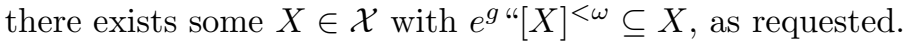

So, in $V[G][H], \mathcal{S}$ is a stationary subset of $\left[\aleph_{\omega+1}\right]^{\omega}, \mathrm{GCH}$ holds, and $\nabla_{S(\mathcal{S})}$ fails.

Now, what would have gone wrong had we forced with $\mathbb{P}^{1}\left(\lambda^{+}\right)$instead of $\mathbb{P}\left(\lambda^{+}\right)$? We know that there exists $\overline{\mathcal{X}}: \bigcup_{\eta<\tau}[\eta]^{\omega} \rightarrow 2$ such that if $g \in{ }^{Z} 2$ and $\left\langle\mathcal{X}_{g}, k_{g}\right\rangle$ is a minimal upper bound of $\left\langle q_{g \mid Z_{n}} \mid n<\omega\right\rangle$, then $\operatorname{dom}\left(\mathcal{X}_{g}\right)=[\tau]^{\omega}$ and $\mathcal{X}_{g} \cap \bigcup_{\eta<\tau}[\eta]^{\omega}=$ $\overline{\mathcal{X}}$. Clearly, there is no way of ensuring that for some $g$ there already exists an $X \in \operatorname{dom}(\overline{\mathcal{X}})$ which is closed under $e^{g}$ and $\overline{\mathcal{X}}(X)=1$. However, this is a density argument, so we may consider extensions of $\bar{X}$.

Now, for all $g$, since $\tau$ is a closure point of $e^{g}$, there indeed exists a cofinal subset $X_{g} \in[\tau]^{\omega}$ which is closed under $e^{g}$, and it is tempting to just take a function $\mathcal{X}:[\tau]^{\omega} \rightarrow 2$ extending $\overline{\mathcal{X}}$ and such that $\mathcal{X}\left(X_{g}\right)=1$, and to consider $\left\langle\mathcal{X}, k_{g}\right\rangle$. So, here is the problem - how do we know that the latter is a legitimate condition? As $\tau \in c_{i}^{k_{g}}$ for all $i \in Z$, we need, in particular, to establish that $x_{i}^{k_{g}} \cap \tau \neq \vec{A}_{i}(\tau)$ for all $i \in Z$. In the above construction, we did so by "throwing in" a countable cofinal subset of $\tau$ to $\mathcal{X}_{0}$, thus forcing that $\tau$ is in $S\left(\lambda^{+}\right)$. This time, we are allowed to throw in only a single cofinal subset of $\tau$ to $\mathcal{X}_{0}$, so we need to throw in a cofinal subset of $\tau$ which is closed under $e^{g}$ for all $g \in{ }^{Z} 2$, at once. But, this turns out to be impossible.

\section{OpEn PROBLEMS}

Let $\lambda$ denote a singular cardinal. Probably the most interesting open question in this area is the following question of Shelah:

Question 1. Does $2^{\lambda}=\lambda^{+}$imply $\diamond_{E_{\mathrm{cf}(\lambda)}^{\lambda+}}$ ? Does GCH imply $\diamond_{E_{\mathrm{cf}(\lambda)}^{\lambda+}}$ ?

By [21] and the fact that $E_{\mathrm{cf}(\lambda)}^{\lambda^{+}}$reflects stationarily often, a negative answer to the above question witnesses the failure of $\square_{\lambda}^{*}$, so large cardinals are necessary. 
Question 2. Suppose $S \subseteq E_{\operatorname{cf}(\lambda)}^{\lambda^{+}}$reflects stationarily often; must $\mathrm{NS}_{\lambda^{+}} \uparrow S$ be non-saturated?

By [15, a negative answer to the above question witnesses the failure of $\square_{\lambda}^{*}$ (actually, of $\mathrm{SAP}_{\lambda}$ ). Note that by [9], $\mathrm{NS}_{\lambda^{+}} \uparrow E_{\mathrm{cf}(\lambda)}^{\lambda^{+}}$is indeed non-saturated. Also note that if one does not require reflection, then by results of Woodin and Foreman (see [6, $\S 8]$ ), $\mathrm{NS}_{\aleph_{\omega+1}} \uparrow S$ can consistently be saturated for some stationary (nonreflecting) $S \subseteq E_{\omega}^{\aleph_{\omega+1}}$.

\section{REFERENCES}

[1] James Cummings. Iterated forcing and elementary embeddings. In Matthew Foreman and Akihiro Kanamori, editors, Handbook of set theory, volume II, pages 775-883. SpringerVerlag, 2010.

[2] Keith J. Devlin and Hàvard Johnsbräten. The Souslin problem. Lecture Notes in Mathematics, Vol. 405. Springer-Verlag, Berlin, 1974.

[3] Todd Eisworth. Successors of Singular Cardinals. Handbook of set theory, pages 1229-1350. Springer, Dordrecht, 2010. MR2768694

[4] Mirna Džamonja and Saharon Shelah. On versions of on cardinals larger than $\aleph_{1}$. Math. Japon., 51(1):53-61, 2000. MR1739051 (2001m:03089)

[5] M. Foreman, M. Magidor, and S. Shelah. Martin's maximum, saturated ideals, and nonregular ultrafilters. I. Ann. of Math. (2), 127(1):1-47, 1988. MR924672 (89f:03043)

[6] Matthew Foreman. Ideals and generic elementary embeddings. In Matthew Foreman and Akihiro Kanamori, editors, Handbook of set theory, volume II, pages 885-1147. SpringerVerlag, 2010.

[7] Matthew Foreman and Menachem Magidor. A very weak square principle. J. Symbolic Logic, 62(1):175-196, 1997. MR.1450520 (98i:03062)

[8] Moti Gitik and Assaf Sharon. On SCH and the approachability property. Proc. Amer. Math. Soc., 136(1):311-320 (electronic), 2008. MR2350418 (2008m:03102)

[9] Moti Gitik and Saharon Shelah. Less saturated ideals. Proc. Amer. Math. Soc., 125(5):15231530, 1997. MR1363421 (97g:03055)

[10] R. Björn Jensen. The fine structure of the constructible hierarchy. Ann. Math. Logic, 4:229308; erratum, ibid. 4:443, 1972. With a section by Jack Silver. MR0309729 (46:8834)

[11] Bernhard König, Paul Larson, and Yasuo Yoshinobu. Guessing clubs in the generalized club filter. Fund. Math., 195(2):177-191, 2007. MR2320769 (2008f:03064)

[12] Kenneth Kunen. Set theory, volume 102 of Studies in Logic and the Foundations of Mathematics. North-Holland Publishing Co., Amsterdam, 1980. An introduction to independence proofs. MR.597342 (82f:03001)

[13] Paul Larson. Separating stationary reflection principles. J. Symbolic Logic, 65(1):247-258, 2000. MR.1782117 (2001k:03094)

[14] Assaf Rinot. A topological reflection principle equivalent to Shelah's strong hypothesis. Proc. Amer. Math. Soc., 136(12):4413-4416, 2008. MR2431057 (2009h:03058)

[15] Assaf Rinot. A relative of the approachability ideal, diamond and non-saturation. J. Symbolic Logic, 75(3):1035-1065. 2010. MR2723781

[16] Saharon Shelah. On successors of singular cardinals. In Logic Colloquium '78 (Mons, 1978), volume 97 of Stud. Logic Foundations Math., pages 357-380. North-Holland, Amsterdam, 1979. MR567680(82d:03079)

[17] Saharon Shelah. Diamonds, uniformization. J. Symbolic Logic, 49(4):1022-1033, 1984. MR7771774 (86g:03083)

[18] Saharon Shelah. Cardinal arithmetic, volume 29 of Oxford Logic Guides. The Clarendon Press Oxford University Press, New York, 1994. Oxford Science Publications. MR 1318912 (96e:03001)

[19] Saharon Shelah. Diamonds. Proc. Amer. Math. Soc., 138(6):2151-2161, 2010. MR2596054

[20] Charles I. Steinhorn and James H. King. The uniformization property for $\aleph_{2}$. Israel J. Math., 36(3-4):248-256, 1980. MR.597452 (82a:03051) 
[21] Martin Zeman. Diamond, GCH and weak square. Proc. Amer. Math. Soc., 138(5):1853-1859, 2010. MR2587470

School of Mathematical Sciences, Tel Aviv University, Tel Aviv 69978, Israel

E-mail address: gitik@post.tau.ac.il

URL: http://www.math.tau.ac.il/〜 gitik

School of Mathematical Sciences, Tel Aviv University, Tel Aviv 69978, Israel URL: http://www.math.tau.ac.il/ rinot

Current address: The Fields Institute for Research in Mathematical Sciences, 222 College Street, Toronto, Ontario, Canada M5T 3J1

E-mail address: assaf@rinot.com

$U R L:$ http://www.assafrinot.com 